\title{
Review
}

\section{The Complex Story of Plant Cyclic Nucleotide-Gated Channels}

\author{
Edwin Jarratt-Barnham, Limin Wang $\mathbb{D}^{\mathrm{D}}$, Youzheng Ning and Julia M. Davies * \\ Department of Plant Sciences, University of Cambridge, Cambridge CB2 3EA, UK; ecj39@cam.ac.uk (E.J.-B.); \\ lw577@cam.ac.uk (L.W.); yn283@cam.ac.uk (Y.N.) \\ * Correspondence: jmd32@cam.ac.uk; Tel.: +44-1223-333-939
}

check for updates

Citation: Jarratt-Barnham, E.; Wang, L.; Ning, Y.; Davies, J.M. The Complex Story of Plant Cyclic Nucleotide-Gated Channels. Int. J. Mol. Sci. 2021, 22, 874. https://doi. org/10.3390/ijms22020874

Received: 11 December 2020

Accepted: 15 January 2021

Published: 16 January 2021

Publisher's Note: MDPI stays neutral with regard to jurisdictional claims in published maps and institutional affiliations.

Copyright: (c) 2021 by the authors. Licensee MDPI, Basel, Switzerland. This article is an open access article distributed under the terms and conditions of the Creative Commons Attribution (CC BY) license (https:/ / creativecommons.org/licenses/by/ $4.0 /)$.

\begin{abstract}
Plant cyclic nucleotide-gated channels (CNGCs) are tetrameric cation channels which may be activated by the cyclic nucleotides (cNMPs) adenosine $3^{\prime}, 5^{\prime}$-cyclic monophosphate (cAMP) and guanosine $3^{\prime}, 5^{\prime}$-cyclic monophosphate (cGMP). The genome of Arabidopsis thaliana encodes 20 CNGC subunits associated with aspects of development, stress response and immunity. Recently, it has been demonstrated that CNGC subunits form heterotetrameric complexes which behave differently from the homotetramers produced by their constituent subunits. These findings have widespread implications for future signalling research and may help explain how specificity can be achieved by CNGCs that are known to act in disparate pathways. Regulation of complex formation may involve cyclic nucleotide-gated channel-like proteins.
\end{abstract}

Keywords: calcium signalling; CaM; calmodulin; cAMP; cGMP; CNGC; cyclic nucleotide-gated channel; CNGCL; cyclic nucleotide-gated channel like

\section{Introduction}

Plant cyclic nucleotide-gated channels (CNGCs) are held to be tetrameric cation channels formed by four subunits which may be activated by the cyclic nucleotide monophosphates (cNMPs) adenosine $3^{\prime}, 5^{\prime}$-cyclic monophosphate (cAMP) and guanosine $3^{\prime}, 5^{\prime}$-cyclic monophosphate (cGMP) [1-10]. The ability of cNMPs to act as signalling molecules in plants has been questioned. However, enhanced detection methods are revealing stimulusinduced increases and the ability to lower cAMP in cellular compartments using a "cAMP sponge" is now allowing the consequences of depletion to be investigated [11]. CNGCs are integral not only to plant nutrition, but also to calcium $\left(\mathrm{Ca}^{2+}\right)$ signalling in development, abiotic stress and immunity [9,10,12-15]. Most research into CNGC contribution to signalling has focused on the $20 \mathrm{cngc}$ loss-of-function mutants in Arabidopsis thaliana (summarised in Table 1) and on the functional characteristics of homotetrameric CNGCs in heterologous expression systems such as Escherichia coli, yeast, Xenopus oocytes and HEK293 cells. The 20 AtCNGC gene sequences [16] have been used to predict that Glycine max has 39 [17], Hordeum vulgare has 9 [7], Nicotiana tabacum has 35 [18], Oryza sativa has 16 [19], Triticum aestioum has 47 [20], Zea mays has 12 [21], Brassica oleracea has 26 [22] and Brassica rapa has 30 CNGCs [23]. Advances made with Arabidopsis may well have implications for crop species.

Table 1. AtCNGCs are involved in diverse signalling pathways ranging from development to stress responses. For each CNGC in Arabidopsis thaliana, the reported physiological or developmental roles are presented, based on phenotyping loss-of-function mutants. Where the roles of two or more CNGCs overlap, it may be postulated that a CNGC complex might form between these subunits if they co-localise. Currently, complex formation has only been investigated in a few of these cases.

\begin{tabular}{ccc}
\hline Gene & Proposed Physiological or Developmental Process & References \\
\hline AtCNGC1 & $\begin{array}{c}\text { Negative regulation of } \mathrm{Pb}^{2+} \text { tolerance; primary root growth; } \\
\text { gravitropism }\end{array}$ & {$[24-26]$} \\
\hline
\end{tabular}


Table 1. Cont.

\begin{tabular}{|c|c|c|}
\hline Gene & Proposed Physiological or Developmental Process & References \\
\hline AtCNGC2 & $\begin{array}{l}\text { Pathogen defence; programmed cell death; nitric oxide } \\
\text { generation; suppression of leaf senescence; flowering time; } \\
\text { thermotolerance (heat and chill); } \mathrm{Ca}^{2+} \text { transport in leaves } \\
\text { and } \mathrm{Ca}^{2+} \text { sensitivity; jasmonic acid-induced } \mathrm{Ca}^{2+} \text { entry }\end{array}$ & [27-39] \\
\hline AtCNGC3 & Germination; salt tolerance; $\mathrm{Na}^{+}$and $\mathrm{K}^{+}$uptake & [40] \\
\hline AtCNGC4 & $\begin{array}{l}\text { Pathogen defence; programmed cell death; flowering time; } \\
\text { thermotolerance (heat and chill); } \mathrm{Ca}^{2+} \text { tolerance }\end{array}$ & {$[27-29,32,38,41]$} \\
\hline AtCNGC5 & $\begin{array}{l}\text { cGMP-activated } \mathrm{Ca}^{2+} \text { entry in guard cells; salt tolerance; } \\
\text { root hair growth; auxin signalling }\end{array}$ & {$[4,42,43]$} \\
\hline AtCNGC6 & $\begin{array}{l}\text { cGMP-activated } \mathrm{Ca}^{2+} \text { entry in guard cells; thermotolerance } \\
\text { (heat); root hair growth; auxin signalling }\end{array}$ & {$[4,43-45]$} \\
\hline AtCNGC7 & Pollen tube growth & {$[46,47]$} \\
\hline AtCNGC8 & Pollen tube growth & {$[46,47]$} \\
\hline AtCNGC9 & Root hair growth; auxin signalling & {$[43,44]$} \\
\hline AtCNGC10 & $\begin{array}{l}\text { Negative regulation of salt tolerance; } \mathrm{K}^{+}, \mathrm{Na}^{+} \text {and } \mathrm{Pb}^{2+} \\
\text { uptake; } \mathrm{K}^{+} \text {homeostasis; negative regulation of } \mathrm{Pb}^{2+} \\
\text { tolerance; regulation of starch granule size; gravitropism; } \\
\text { flowering time; hypocotyl elongation }\end{array}$ & {$[25,48,49]$} \\
\hline AtCNGC11 & $\begin{array}{l}\text { Pathogen defence; programmed cell death; } \mathrm{Pb}^{2+} \text { and } \mathrm{Cd}^{2+} \\
\text { uptake; } \mathrm{Pb}^{2+} \text { tolerance; negative regulation of } \mathrm{Cd}^{2+} \\
\text { tolerance }\end{array}$ & {$[15,25,50-53]$} \\
\hline AtCNGC12 & Pathogen defence; programmed cell death & {$[15,50-53]$} \\
\hline AtCNGC13 & $\mathrm{Pb}^{2+}$ uptake; negative regulation of $\mathrm{Pb}^{2+}$ tolerance & [25] \\
\hline AtCNGC14 & Root hair growth; gravitropism; auxin signalling & {$[43,44,54-57]$} \\
\hline AtCNGC15 & $\mathrm{Pb}^{2+}$ and $\mathrm{Cd}^{2+}$ uptake; $\mathrm{Pb}^{2+}$ tolerance; root development & {$[25,58]$} \\
\hline AtCNGC16 & $\begin{array}{l}\text { Heat and drought tolerance in pollen; negative regulation of } \\
\qquad \mathrm{Cd}^{2+} \text { tolerance }\end{array}$ & {$[25,59]$} \\
\hline AtCNGC17 & Growth regulation; salt tolerance & {$[42,60]$} \\
\hline AtCNGC18 & Pollen tube growth and guidance & {$[6,46,61-63]$} \\
\hline AtCNGC19 & $\begin{array}{l}\text { Response to salt; } \mathrm{Pb}^{2+} \text { and } \mathrm{Cd}^{2+} \text { uptake; negative regulation } \\
\text { of } \mathrm{Pb}^{2+} \text { tolerance; herbivory response; pathogen defence; } \\
\text { endophyte response; regulating cell death }\end{array}$ & {$[25,64-67]$} \\
\hline AtCNGC20 & Response to salt; pathogen defence; regulating cell death & {$[65,67]$} \\
\hline
\end{tabular}

There is increasing evidence to suggest that CNGCs form heterotetrameric complexes which may have unique functional characteristics, compared to homotetrameric channels $[9,10,27-29,44,46]$. These may help facilitate the generation of stimulus-specific $\mathrm{Ca}^{2+}$ signatures (as monophasic, biphasic or oscillatory increases in this second messenger in a given cellular compartment) that could be decoded by specific complements of $\mathrm{Ca}^{2+}$-binding proteins to cause a stimulus-specific response $[9,10]$. These discoveries, in combination with recent advances in the model of CNGC structure, have major implications for our understanding of CNGC function and generate new areas for future research. Here, precedents for diverse channel subunit interactions are reviewed, with consideration of in vivo factors that may determine the subunits of CNGC complexes. Additionally, regulatory diversity of AtCNGC subunits is reviewed as a critical determinant of heterotetrameric channel function, with the possibility that CNGC-like proteins (if present) may restrict complex formation. 


\section{Heteromeric Channel Complexes Also Occur in Plants}

It has been known for some time that animal cyclic nucleotide-gated (CNG) channels form heteromeric complexes and that the combinations of these subunits define the functional characteristics of the channel [68-71]. In mammalian retinal phototransduction, CNG channels in rod cells are formed by three subunits of CNGA1 and one CNGB1 subunit, with the C-terminal leucine zipper region of the CNGA1 subunits interacting to set the stoichiometry $[70,71]$. In contrast, in cone cells, the channel is formed by three subunits of CNGA3 and one CNGB3 subunit. It is held that the CNGB subunits "fine tune" channel behaviour by regulating opening/closing kinetics, affinity for cyclic nucleotides and ability to be regulated by $\mathrm{Ca}^{2+}$ [70]. Precedents for heterotetrameric channel assembly in plants have come from members of the Shaker voltage-dependent $\mathrm{K}^{+}$channel family, underpinning $\mathrm{K}^{+}$uptake and distribution. Interactions amongst Shaker channel subunits are dependent on specific regions of the cytosolic C-terminal domain (CT) and specific subunits may have inhibitory effects on the overall channel complex [72-76]. A breakthrough study on Medicago truncatula nodulation nuclear signalling revealed an interaction between the $\mathrm{K}^{+}$ channel DMI1 (Does not Make Infections 1) and MtCNGC15s, probably to enable voltage change mediated by DMI1 to promote MtCNGC15s opening and $\mathrm{Ca}^{2+}$ flux [77]. This opens up the possibility of interaction between entirely different channel families. Although there are no reports of interaction between $\mathrm{K}^{+}$channels and CNGCs in Arabidopsis or other plants, there is now a good body of evidence that Arabidopsis CNGC subunits (AtCNGCs) also form heterotetrameric channels within the family. CNGC-CNGC interactions have also been proposed in Zea mays and the moss Physcomitrella patens [21,28].

Bifluorescence complementation (BiFC) in Nicotiana benthamiana has shown interactions occur in planta between AtCNGC2 and AtCNGC4 [27], between AtCNGC8 and AtCNGC18 [46], between AtCNGC7 and AtCNGC18 [46], and between AtCNGC19 and AtCNGC20 [67]. BiFC analyses also suggest that AtCNGC6, AtCNGC9 and AtCNGC14 interact with each other (AtCNGC6/9, AtCNGC6/14, AtCNGC9/14) [44]. To demonstrate that $\mathrm{BiFC}$ signals are produced by heterotetrameric complexes, instead of clustered homotetramers, Pan et al. analysed single-molecule fluorescence to determine that co-expression of AtCNGC7 or AtCNGC8 with AtCNGC18 resulted in heterotetrameric AtCNGC7/18 or AtCNGC8/18 complexes with a 2:2 stoichiometry [46]. This was an important breakthrough in establishing tetramer formation. Prior to this, the need for four CNGC subunits to combine to make a channel had been assumed by analogy with animal and bacterial channels, then supported by molecular modelling [78] (see Section 4 on channel structure). The range of subunit interactions found to date shows that they are not limited to co-members of the five phylogenetic groups within the family (I, II, III, IV-A and IV-B; Figure 1 [16-19,21-23,79]) but can occur across groups. Therefore, CNGC-CNGC interactions are isoform specific but are not restricted to closely related CNGC isoforms. In contrast, AtCNGC16 and AtCNGC18 interactions have not been observed [46], demonstrating that members of the same group (in this case group III) may not work together and that phylogeny alone may not be a useful tool in predicting interactions. Rather, a consideration of co-localisation is needed. 


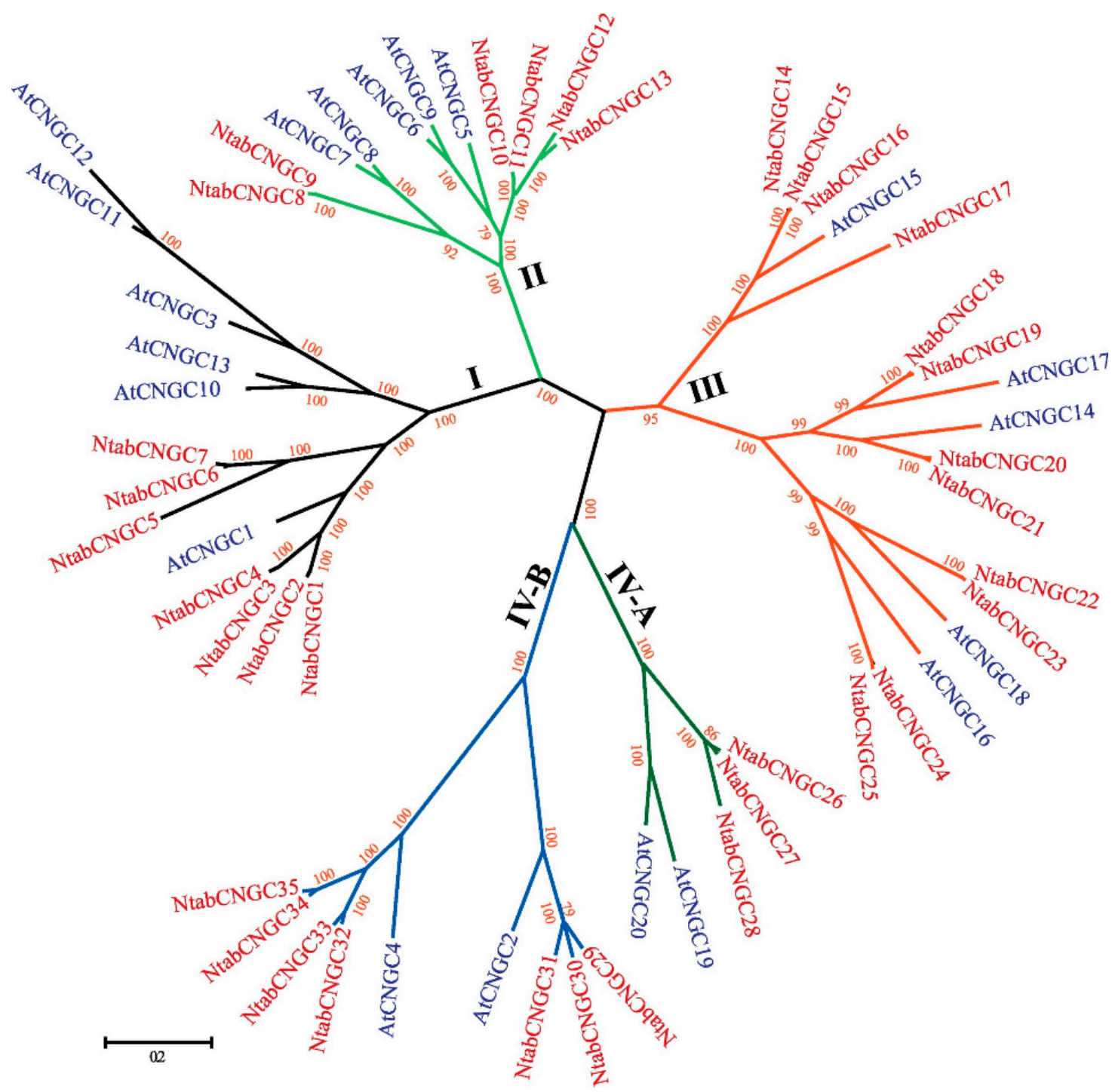

Figure 1. The phylogenetic relationship between CNGCs of Arabidopsis thaliana and Nicotiana tabacum. Multiple sequence alignment was carried out with the MUSCLE program and MEGA 6.0 used to generate the tree using the Jones-TaylorThornton (JTT) model. Bootstrap values from 1000 repeats are shown. Figure reproduced from Nawaz et al. 2019 [18], with permission of the Publisher.

\section{Complex Formation Would Depend on the Co-Localisation and Relative Abundance of CNGC Isoforms}

Whether a CNGC is present in the plasma membrane or an endomembrane (as a homotetramer or in a heterotetrameric complex) has consequences for the generation of a signal-specific $\left[\mathrm{Ca}^{2+}\right]_{\mathrm{cyt}}$ signature. It is envisaged that CNGC plasma membrane localisation would play a part in signature initiation driven by receptors in that membrane whilst CNGCs in the tonoplast, for example, would be downstream and acting to amplify the $\left[\mathrm{Ca}^{2+}\right]_{\text {cyt }}$ signal. Most CNGCs are believed to localise to the plasma membrane, and this has been reported for many AtCNGCs, including AtCNGC2 [33], AtCNGC3 [40], AtCNGC6 [44], AtCNGC7 [46], AtCNGC8 [46], AtCNGC9 [44], AtCNGC10 [48,80], AtCNGC11 [51], AtCNGC12 [51,81], AtCNGC14 [44], AtCNGC17 [60], AtCNGC18 [46,62,63], AtCNGC19 [66] and AtCNGC20 [82]. AtCNGC5 is reported to localise in microdomains at the periphery of $N$. benthamiana protoplasts when expressed heterologously [4]. It must be noted, however, that in contradiction of the findings by Meena et al. [66] and Fischer et al. [82], Yuen and Christopher reported that AtCNGC19 and AtCNGC20 localised to the vacuolar membrane, not the plasma membrane [83]. Similarly, Chang et al. found 
AtCNGC7, AtCNGC8 and AtCNGC16 to localise to endomembrane in pollen tubes, as opposed to the plasma membrane [61]. In silico predictions suggest that 11 of 12 Zea mays CNGCs could localise to the plasma membrane [21]. The importance of localisation is exemplified by the positioning of nuclear CNGCs to enable nuclear $\mathrm{Ca}^{2+}$ "spiking" in symbiosis signalling [77]. MtCNGC15a, MtCNGC15b and MtCNGC15c of M. truncatula localise to the nuclear envelope, with 14 of $21 \mathrm{MtCNGCs}$ predicted to contain nuclear localisation sequences [77]. Recently, AtCNGC15 was also found to localise to the nuclear membrane and to be involved in root development [58]. From these results, it is clear that many CNGCs could co-localise, which would facilitate extensive CNGC-CNGC subunit interactions, including the formation of heterotetrameric complexes.

These interactions will necessarily be dependent on the co-expression of CNGCs within individual cells. Transcriptomic data indicate that many CNGCs (23 of 35 in N. tabacum [18]) are expressed throughout the plant. Expression levels, however, vary greatly, suggesting that many CNGCs have tissue-specific or cell-specific functions [4,18,21-23,44,47]. Expression of an individual CNGC can vary with development and growth conditions. For example, leaf expression of AtCNGC3 increases with leaf age and lessens when the plant is grown in soil rather than on an agar plate [40]. CNGC promotors have been found to contain elements associated with responses to abscisic acid, auxin, ethylene, gibberellin, salicylic acid and methyl-jasmonate [17-21], and CNGC transcript levels are highly sensitive to abiotic and biotic stressors [17-20,22,23,79]. Notably, it has also been found that Ziziphus jujube CNGC2 expression is rapidly induced by application of cAMP to callus [79], potentially providing a mechanism for priming signalling pathways involving ZjCNGC2. In silico analysis has also predicted that NtabCNGC expression may be regulated extensively by micro-RNAs and cis-acting regulatory elements [18]. Consequently, the abundance of different CNGC isoforms in each cell type is likely to be variable, and dependent on environmental conditions, which may result in the formation of different CNGC heterotetramers in different cell types.

A further consideration is that studies typically measure transcript levels from entire organs and may lack sufficient spatial resolution to detect low, cell-specific expression [17-19,22,23,44]. AtCNGC5, AtCNGC6, AtCNGC9 and AtCNGC14 have been implicated in root hair growth and their transcripts are abundant in roots $[43,44,54-57]$. However, CNGCpromotor::GUS fusions of AtCNGC6, AtCNGC9 and AtCNGC14 also suggest that these $A t C N G C s$ are expressed throughout the plant [44]. AtCNGC6 expression is predicted in root, shoot, leaf and guard cells [4,44,45]. AtCNGC9 expression is predicted in root hairs and guard cells but not leaves or shoots, and AtCNGC14 expression is predicted in roots, shoots and the leaf [44]. As stated earlier, BiFC suggests that AtCNGC6, AtCNGC9 and AtCNGC14 may form a range of heterotetrameric complexes with each other [44]. From these data, it may be predicted that different complexes form in different cell types depending on the expression patterns of the interacting partners. Consequently, the signalling function of these AtCNGCs may be cell type specific. Notably, AtCNGC6 has been implicated not only in root hair growth polarity but also in thermotolerance and cGMP-induced $\mathrm{Ca}^{2+}$ influx in guard cells (in which it could partner AtCNGC5) $[4,28,44]$. Additionally, whilst AtCNGC9 has so far only been implicated in root hair growth polarity, its expression in guard cells would suggest a specific activity there as part of a complex with AtCNGC6. Both guard cell and mesophyll plasma membrane contain $\mathrm{Ca}^{2+}$ channels that are activated by cyclic nucleotides [84]. It is, therefore, possible that the formation of different CNGC complexes with unique functional characteristics in different cell types helps permit stimulus-specific signalling in the diverse pathways that AtCNGC6 and other AtCNGCs work in (Table 1).

The relative abundance of CNGC subunits is also likely to be a key determinant of CNGC complex formation and function. Yoshioka et al. discovered that the phenotypes associated with the cpr22 mutant (a gene fusion between AtCNGC11 and AtCNGC12) could be supressed by overexpression of $A t C N G C 12$, and it was proposed that this was due to disruption of heterotetrameric complex formation [53]. Notably, AtCNGC6 transcripts 
are $>4.5$ times more abundant than AtCNGC9 transcripts in guard cells [4]. If these two CNGCs were to form heterotetrameric complexes (as suggested) and transcript abundance were proportional to protein abundance, then excess AtCNGC6 subunits must either form homotetramers, be degraded, sequestered in the membrane, or interact with additional AtCNGC subunits. Following a study of AtCNGC2 homologues in P. patens by Finka et al., such an interaction between AtCNGC2 and AtCNGC6 has been proposed for heat signalling [28]. In pollen tube growth, the ratio of AtCNGC18 to AtCNGC 8 expression is expected to determine the proportion of AtCNGC8/18 complexes relative to AtCNGC8 or AtCNGC18 homotetramers, leading to changes in cell permeability [46]. When expressed singly in Xenopus oocytes, AtCNCG18 forms a constitutively voltage-dependent, $\mathrm{Ca}^{2+}$-permeable channel but AtCNGC8 is electrically silent (as is AtCNGC7). Equimolar co-expression of AtCNGC18 and AtCNGC8 (presumably with heterotetramer formation) resulted in greatly reduced $\mathrm{Ca}^{2+}$ influx compared to AtCNGC18 expression alone [46]. Pan et al. proposed that, by recruiting AtCNGC18 into heterotetrameric AtCNGC8/18 complexes, AtCNGC8 represses AtCNGC18 activity [46]. Consequently, whilst the overexpression of AtCNGC18 in $A$. thaliana disrupts $\mathrm{Ca}^{2+}$-dependent pollen tube growth, potentially by forming deregulated AtCNGC18 homotetramers, this phenotype can be rescued by overexpressing AtCNGC8, presumably by recruiting AtCNGC18 subunits from homotetramers to generate heterotetramers [46]. However, it should be noted that Gao et al. reported that expression of $A t C N G C 8$ or AtCNGC7 in HEK293T cells produced constitutively voltage-dependent, $\mathrm{Ca}^{2+}$-permeable channels which were activated further by addition of $8 \mathrm{Br}-\mathrm{cNMPs}$ [5]. This contradiction may be caused by the different bathing solutions used in each study or the use of different heterologous expression systems. Examples of key findings from transport studies on heterologously expressed CNGC genes are shown in Table 2. This further shows that the choice of expression system may have an effect on the outcome. For example, $\mathrm{K}^{+}$ selectivity over $\mathrm{Na}^{+}$of AtCNGC2 was greater in HEK293 cells than in Xenopus oocytes [2]. Nevertheless, AtCNGC7/18, and AtCNGC8/18 complexes appear to form spontaneously in a heterologous expression system, yielding different transport characteristics to the homomeric forms [46]. If single-molecule fluorescence were to confirm the existence of an AtCNGC2/4 complex, as well as complexes amongst the AtCNGC6, AtCNGC9 and AtCNGC14 triad and AtCNGC19/20 couple as predicted by BiFC [27,44,67], then it is likely that CNGC complexes could be widespread if co-localisation permits.

Table 2. Examples of transport characteristics and response to cNMPs of CNGCs when either heterologously expressed or present in native membrane. Most studies have focused on the CNGCs of Arabidopsis thaliana (AtCNGC). However, there are also studies reporting the activity of CNGCs in Physcomitrella patens (PpCNGC), Hordeum vulgare (HvCNGC), Oryza sativa (OsCNGC) and Medicago truncatula (MtCNGC) as detailed below. "Whole cell" refers to the recording configuration in which channel activity is captured from effectively the entire plasma membrane. "Inside-out patch" refers to the configuration in which the cytosolic face of a membrane patch (held in the electrode tip) faces the bathing medium. "Cell attached" refers to the configuration in which the membrane patch (held in the electrode tip) remains undetached from the remaining membrane.

\begin{tabular}{ccccc}
\hline CNGCs & System & Tested Cations & Tested with cNMPs? & Results \\
\hline AtCNGC1 & $\begin{array}{l}\text { HEK293- } \\
\text { whole cell }\end{array}$ & $\begin{array}{c}\text { Tested } \mathrm{K}^{+} \text {and } \\
\mathrm{Na}^{+} \text {conductance }\end{array}$ & Yes & $\begin{array}{c}\text { Application of } 100 \mu \mathrm{M} \\
\text { db-cAMP stimulated } \\
\text { AtCNGC1 } \mathrm{K}^{+} \text {and } \mathrm{Na}^{+} \\
\end{array}$
\end{tabular}


Table 2. Cont.

\begin{tabular}{|c|c|c|c|c|c|}
\hline CNGCs & System & Tested Cations & Tested with cNMPs? & Results & References \\
\hline AtCNGC1 & Yeast & $\begin{array}{c}\text { Tested } \mathrm{Ca}^{2+} \\
\text { uptake }\end{array}$ & No & $\begin{array}{l}\text { In the presence of yeast } \\
\text { pheromone } \alpha \text { factor, } \\
\text { AtCNGC1 in a Ca }{ }^{2+} \\
\text { uptake-deficient yeast mutant } \\
\text { increased colony growth, } \\
\text { indirectly demonstrating } \mathrm{Ca}^{2+} \\
\text { conduction. }\end{array}$ & [24] \\
\hline $\begin{array}{l}\text { AtCNGC1, } \\
\text { AtCNGC2, } \\
\text { AtCNGC4 }\end{array}$ & Yeast & Tested $\mathrm{K}^{+}$uptake & Yes & $\begin{array}{l}\text { Addition of } 100 \mu \mathrm{M} \mathrm{db}-\mathrm{cAMP} \\
\text { stimulated growth of a } \mathrm{K}^{+} \\
\text {uptake-deficient yeast mutant } \\
\text { expressing AtCNGC1, } \\
\text { AtCNGC2, and AtCNGC4. }\end{array}$ & {$[86]$} \\
\hline $\begin{array}{l}\text { AtCNGC1, } \\
\text { AtCNGC2, } \\
\text { AtCNGC4 }\end{array}$ & Yeast & $\begin{array}{l}\text { Tested } \mathrm{K}^{+} \text {and } \\
\mathrm{Ca}^{2+} \text { uptake }\end{array}$ & Yes & $\begin{array}{l}\text { AtCNGC1M2 (deletion in } \\
\text { C-terminal domain) } \\
\text { expression in a } \mathrm{Ca}^{2+} \text { uptake } \\
\text { yeast mutant resulted in } \\
\text { growth, indicating } \mathrm{Ca}^{2+} \\
\text { permeability of AtCNGC1. } \\
\text { Expression of } A t C N G C 2 \text { and } \\
\text { AtCNGC4 enhanced growth of } \\
\text { a } \mathrm{K}^{+} \text {uptake-deficient yeast } \\
\text { mutant. Application of } 100 \\
\mu \mathrm{M} \text { db-cAMP increased } \\
\text { growth of yeast mutant } \\
\text { transformed with } \\
\text { AtCNGC1M2. }\end{array}$ & [87] \\
\hline AtCNGC2 & Yeast & Tested $\mathrm{K}^{+}$uptake & Yes & $\begin{array}{c}\text { In the presence of } 10 \mu \mathrm{M} \\
\text { db-cAMP or db-cGMP, } \\
\text { transfection with } A t C N G C 2 \\
\text { enhanced growth of a } \mathrm{K}^{+} \\
\text {uptake-deficient yeast mutant. }\end{array}$ & [1] \\
\hline AtCNGC2 & $\begin{array}{c}\text { Xenopus } \\
\text { oocytes- } \\
\text { whole cell }\end{array}$ & $\begin{array}{c}\text { Tested } \mathrm{K}^{+} \\
\text {conductance }\end{array}$ & Yes & $\begin{array}{c}\text { Application of } 10 \mu \mathrm{M} \\
\text { db-cAMP stimulated } \\
\text { AtCNGC2 } \mathrm{K}^{+} \text {conductance. } \\
\text { No } \mathrm{K}^{+} \text {conductance was } \\
\text { observed in the absence of } \\
\text { db-cNMPs. }\end{array}$ & [1] \\
\hline AtCNGC2 & $\begin{array}{c}\text { Xenopus } \\
\text { oocytes- } \\
\text { whole cell }\end{array}$ & $\begin{array}{l}\text { Tested } \mathrm{K}^{+}, \mathrm{Na}^{+}, \\
\mathrm{Li}^{+}, \mathrm{Cs}^{+} \text {and } \mathrm{Rb}^{+} \\
\text {conductance }\end{array}$ & Yes & $\begin{array}{l}\text { In the presence of } 100 \mu \mathrm{M} \\
\text { db-cAMP, AtCNGC2 } \\
\text { conducted } \mathrm{K}^{+}, \mathrm{Li}^{+}, \mathrm{Cs}^{+} \text {and } \\
\mathrm{Rb}^{+} . \mathrm{Na}^{+} \text {conductance was } \\
\text { significantly less. No data } \\
\text { were reported concerning } \\
\text { conductance in the absence of } \\
\text { db-cAMP. }\end{array}$ & [2] \\
\hline AtCNGC2 & $\begin{array}{l}\text { Xenopus } \\
\text { oocytes- } \\
\text { inside-out } \\
\text { patch }\end{array}$ & $\begin{array}{c}\text { Tested } \mathrm{K}^{+} \\
\text {conductance }\end{array}$ & Yes & $\begin{array}{l}\text { Application of } 100 \mu \mathrm{M} \text { cAMP } \\
\text { stimulated AtCNGC2 K+ } \\
\text { conductance. No K } \\
\text { conductance was observed in } \\
\text { the absence of db-cAMP. }\end{array}$ & [2] \\
\hline
\end{tabular}


Table 2. Cont.

\begin{tabular}{|c|c|c|c|c|c|}
\hline CNGCs & System & Tested Cations & Tested with cNMPs? & Results & References \\
\hline AtCNGC2 & $\begin{array}{l}\text { Xenopus } \\
\text { oocytes- } \\
\text { inside-out } \\
\text { patch }\end{array}$ & $\begin{array}{c}\text { Tested } \mathrm{K}^{+} \text {and } \\
\mathrm{Na}^{+} \text {conductance }\end{array}$ & Yes & $\begin{array}{l}\text { Application of } 100 \mu \mathrm{M} \mathrm{cAMP} \\
\text { stimulated AtCNGC2 } \mathrm{K}^{+} \\
\text {conductance, but not } \mathrm{Na}^{+} \\
\text {conductance. No } \mathrm{K}^{+} \text {or } \mathrm{Na}^{+} \\
\text {conductance was observed in } \\
\text { the absence of cAMP. } \\
\text { Mutation of N416 and D417 in } \\
\text { the pore resulted in } \mathrm{Na}^{+} \\
\text {conductance similar to } \mathrm{K}^{+} \\
\text {conductance. }\end{array}$ & [85] \\
\hline AtCNGC2 & $\begin{array}{l}\text { HEK293- } \\
\text { whole cell and } \\
\text { inside-out } \\
\text { patch }\end{array}$ & $\begin{array}{c}\text { Tested } \mathrm{K}^{+} \text {and } \\
\mathrm{Na}^{+} \text {conductance }\end{array}$ & Yes & $\begin{array}{c}\text { Application of } 100 \mu \mathrm{M} \\
\mathrm{db}-\mathrm{cAMP} \text { stimulated } \\
\text { AtCNGC2 } \mathrm{K}^{+} \text {conductance, } \\
\text { but not } \mathrm{Na}^{+} \text {conductance. No } \\
\mathrm{K}^{+} \text {or } \mathrm{Na}^{+} \text {conductance was } \\
\text { observed in the absence of } \\
\text { db-cAMP. Mutation of N416 } \\
\text { and D417 in the pore region } \\
\text { resulted in } \mathrm{Na}^{+} \text {conductance } \\
\text { similar to } \mathrm{K}^{+} \text {conductance. }\end{array}$ & {$[2,85]$} \\
\hline AtCNGC2 & $\begin{array}{l}\text { Guard cell } \\
\text { protoplasts- } \\
\text { whole cell }\end{array}$ & $\begin{array}{c}\text { Tested } \mathrm{Ba}^{2+} \\
\text { conductance }(\text { as } \\
\text { a proxy for } \mathrm{Ca}^{2+} \text { ) }\end{array}$ & Yes & $\begin{array}{c}\text { Application of } 1 \mathrm{mM} \\
\text { db-cAMP stimulated } \\
\text { AtCNGC2-dependent } \mathrm{Ca}^{2+} \\
\text { conductance. }\end{array}$ & [30] \\
\hline AtCNGC2 & $\begin{array}{l}\text { HEK293T- } \\
\text { whole cell }\end{array}$ & $\begin{array}{l}\text { Tested } \mathrm{Ca}^{2+} \\
\text { conductance }\end{array}$ & Yes & $\begin{array}{c}\text { Application of } 200 \mu \mathrm{M} \\
\text { 8Br-cAMP stimulated } \\
\text { AtCNGC2 } \mathrm{Ca}^{2+} \text { conductance. } \\
\text { No data were reported } \\
\text { concerning } \mathrm{Ca}^{2+} \text { conductance } \\
\text { in the absence of db-cAMP. }\end{array}$ & [38] \\
\hline $\begin{array}{l}\text { AtCNGC2, } \\
\text { AtCNGC4 }\end{array}$ & $\begin{array}{l}\text { Mesophyll cell } \\
\text { protoplasts- } \\
\text { whole cell }\end{array}$ & $\begin{array}{c}\text { Tested } \mathrm{Ba}^{2+} \\
\text { conductance }(\text { as } \\
\left.\text { a proxy for } \mathrm{Ca}^{2+}\right)\end{array}$ & No & $\begin{array}{l}\text { Wild-type mesophyll cell } \\
\text { protoplasts conducted } \mathrm{Ca}^{2+} \text { in } \\
\text { response to } \mathrm{H}_{2} \mathrm{O}_{2} \text { or flg22. } \\
\mathrm{Ca}^{2+} \text { conductance was lost in } \\
\text { Atcngc2 or Atcngc4 loss-of } \\
\text { function mutants, as well as } \\
\text { the Atcngc2 Atcngc4 double } \\
\text { mutant. }\end{array}$ & [29] \\
\hline $\begin{array}{l}\text { AtCNGC2, } \\
\text { AtCNGC4 }\end{array}$ & $\begin{array}{l}\text { Xenopus } \\
\text { oocytes- } \\
\text { whole cell }\end{array}$ & $\begin{array}{l}\text { Tested } \mathrm{Ca}^{2+} \\
\mathrm{Mg}^{2+}, \mathrm{Ba}^{2+}, \mathrm{Sr}^{2+} \\
\mathrm{K}^{+} \text {and } \mathrm{Na}^{+} \\
\text {conductance }\end{array}$ & No & $\begin{array}{l}\text { Independently, AtCNGC2 or } \\
\text { AtCNGC4 did not conduct } \\
\mathrm{Ca}^{2+} \text { in the absence of cNMPs. } \\
\mathrm{Co} \text {-expression of } A t C N G C 2 \\
\text { and } A t C N G C 4 \text { produced } \mathrm{Ca}^{2+}, \\
\mathrm{Sr}^{2+}, \mathrm{Ba}^{2+} \text { and } \mathrm{K}^{+} \text {-permeable } \\
\left(\mathrm{Na}^{+} \text {and } \mathrm{Mg}^{2+} \text {-impermeable) }\right. \\
\text { channels in the absence of } \\
\text { cNMPs. }\end{array}$ & [29] \\
\hline AtCNGC3 & Yeast & $\begin{array}{l}\text { Tested } \mathrm{Na}^{+} \text {and } \\
\mathrm{K}^{+} \text {uptake }\end{array}$ & No & $\begin{array}{c}\text { Yeast expressing } \mathrm{CNGC3} \\
\text { accumulated more } \mathrm{Na}^{+} \text {and } \\
\mathrm{K}^{+} \text {, suggesting a pathway for } \\
\mathrm{Na}^{+} \text {and } \mathrm{K}^{+} \text {transport. }\end{array}$ & [40] \\
\hline
\end{tabular}


Table 2. Cont.

\begin{tabular}{|c|c|c|c|c|c|}
\hline CNGCs & System & Tested Cations & Tested with cNMPs? & Results & References \\
\hline AtCNCG4 & $\begin{array}{l}\text { Xenopus } \\
\text { oocytes- } \\
\text { inside-out } \\
\text { patch }\end{array}$ & $\begin{array}{l}\text { Tested } \mathrm{K}^{+}, \mathrm{Na}^{+} \\
\text {and } \mathrm{Cs}^{+} \\
\text {conductance }\end{array}$ & Yes & $\begin{array}{l}\text { Application of } 500 \mu \mathrm{M} \mathrm{cAMP} \\
\text { or cGMP stimulated } \\
\text { AtCNGC4 } \mathrm{K}^{+}, \mathrm{Na}^{+} \text {and } \mathrm{Cs}^{+} \\
\text {conductance. Compared to } \\
\mathrm{K}^{+} \text {, outward conductance of } \\
\mathrm{Cs}^{+} \text {was significantly lower. } \\
\mathrm{No}^{-} \text {conduction of } \mathrm{K}^{+}, \mathrm{Na}^{+} \text {or } \\
\mathrm{Cs}^{+} \text {was observed in the } \\
\text { absence of cNMPs. }\end{array}$ & [41] \\
\hline $\begin{array}{l}\text { AtCNGC5, } \\
\text { AtCNGC6 }\end{array}$ & $\begin{array}{l}\text { Guard cell } \\
\text { protoplasts- } \\
\text { whole cell }\end{array}$ & $\begin{array}{l}\text { Tested } \mathrm{Mg}^{2+} \\
\mathrm{Ba}^{2+} \text { and } \mathrm{Ca}^{2+} \\
\text { conductance }\end{array}$ & Yes & $\begin{array}{c}\text { Application of } 500 \mu \mathrm{M} \\
\text { 8Br-cGMP stimulated } \mathrm{Mg}^{2+} \text {, } \\
\mathrm{Ca}^{2+} \text { and } \mathrm{Ba}^{2+} \text { conductance. } \\
\mathrm{Mg}^{2+} \text { conductance was lost in } \\
\text { Atcngc5 Atcngc6 double } \\
\text { mutants. AtCNGC1, } \\
\text { AtCNGC2 and AtCNGC20 } \\
\text { did not appear to contribute } \\
\text { to these guard cell } \\
\text { 8Br-cGMP-activated currents. }\end{array}$ & [4] \\
\hline $\begin{array}{l}\text { AtCNGC5, } \\
\text { AtCNGC6 }\end{array}$ & $\begin{array}{l}\text { HEK293T- } \\
\text { whole cell }\end{array}$ & $\begin{array}{l}\text { Tested } \mathrm{Ca}^{2+} \text { and } \\
\mathrm{Na}^{+} \text {conductance }\end{array}$ & No & $\begin{array}{c}\text { HEK293 cells expressing } \\
\text { CNGC5 or CNGC } 6 \text { displayed } \\
\text { inward currents carried by } \\
\mathrm{Ca}^{2+}, \text { not } \mathrm{Na}^{+} .\end{array}$ & [43] \\
\hline AtCNGC6 & $\begin{array}{l}\text { Root } \\
\text { protoplasts- } \\
\text { whole cell }\end{array}$ & $\begin{array}{l}\text { Tested } \mathrm{Ca}^{2+} \\
\text { conductance }\end{array}$ & Yes & $\begin{array}{c}\text { Application of } 50 \mu \mathrm{M} \\
\text { db-cAMP stimulated } \\
\text { AtCNGC6-dependent } \mathrm{Ca}^{2+} \\
\text { conductance, application of a } \\
\text { phosphodiesterase inhibitor } \\
\text { also stimulated } \mathrm{Ca}^{2+} \\
\text { conductance. }\end{array}$ & [45] \\
\hline $\begin{array}{l}\text { AtCNGC7, } \\
\text { AtCNGC8 }\end{array}$ & $\begin{array}{l}\text { Xenopus } \\
\text { oocytes- } \\
\text { whole cell }\end{array}$ & $\begin{array}{l}\text { Tested } \mathrm{Ca}^{2+} \\
\text { conductance }\end{array}$ & No & $\begin{array}{c}\text { AtCNGC7 or AtCNGC8 } \mathrm{Ca}^{2+} \\
\text { conductivity was not } \\
\text { observed in the absence of } \\
\text { cNMPs. }\end{array}$ & [46] \\
\hline $\begin{array}{c}\text { AtCNGC7, } \\
\text { AtCNGC8, } \\
\text { AtCNGC9, } \\
\text { AtCNGC10, } \\
\text { AtCNGC16 } \\
\text { and } \\
\text { AtCNGC18 }\end{array}$ & $\begin{array}{l}\text { HEK293T- } \\
\text { whole cell }\end{array}$ & $\begin{array}{l}\text { Tested } \mathrm{Ca}^{2+} \text { and } \\
\mathrm{K}^{+} \text {conductance }\end{array}$ & Yes & $\begin{array}{c}\text { Application of } 100 \mu \mathrm{M} \\
\text { 8Br-cAMP or } 100 \mu \mathrm{M} \\
\text { 8Br-cGMP stimulated } \\
\text { AtCNGC7, AtCNGC8, } \\
\text { AtCNGC9, AtCNGC10, } \\
\text { AtCNGC16 and AtCNGC18 } \\
\mathrm{Ca}^{2+} \text { conductance. } \mathrm{Ca}^{2+} \\
\text { conductance did not require } \\
\text { 8Br-cNMP application. No } \\
\text { significant } \mathrm{K}^{+} \text {conductance } \\
\text { reported. }\end{array}$ & [6] \\
\hline AtCNGC10 & $\begin{array}{l}\text { E. coli and } \\
\text { yeast }\end{array}$ & Tested $\mathrm{K}^{+}$uptake & Yes & $\begin{array}{l}\text { AtCNGC10 complemented } E \text {. } \\
\text { coli and yeast } \mathrm{K}^{+} \text {uptake } \\
\text { mutants. In E. coli, } \\
\text { co-expression of AtCNGC10 } \\
\text { and CaM inhibited cell } \\
\text { growth, but cGMP overcame } \\
\text { this. }\end{array}$ & [88] \\
\hline
\end{tabular}


Table 2. Cont

\begin{tabular}{|c|c|c|c|c|c|}
\hline CNGCs & System & Tested Cations & Tested with cNMPs? & Results & References \\
\hline AtCNGC10 & $\begin{array}{l}\text { HEK293- } \\
\text { whole cell }\end{array}$ & $\begin{array}{c}\text { Tested } \mathrm{K}^{+} \\
\text {conductance }\end{array}$ & Yes & $\begin{array}{c}\text { In the presence of } 100 \mu \mathrm{M} \\
\mathrm{db}-\mathrm{cGMP}, \text { AtCNGC10 } \\
\text { conducted } \mathrm{K}^{+} \text {. No data were } \\
\text { reported concerning } \\
\text { conductance in the absence of } \\
\text { db-cAMP. }\end{array}$ & [80] \\
\hline AtCNGC10 & Yeast & $\begin{array}{l}\text { Tested } \mathrm{K}^{+} \text {and } \\
\mathrm{Na}^{+} \text {uptake }\end{array}$ & No & $\begin{array}{l}\text { AtCNGC10-transformed yeast } \\
\text { accumulated more } \mathrm{Na}^{+} \text {in the } \\
\text { presence of } 20 \mathrm{mM} \mathrm{NaCl} \text {. } \\
\text { Expression rescued growth of } \\
\mathrm{K}^{+} \text {uptake-deficient yeast. }\end{array}$ & [49] \\
\hline $\begin{array}{l}\text { AtCNGC11, } \\
\text { AtCNGC12 }\end{array}$ & Yeast & Tested $\mathrm{K}^{+}$uptake & Yes & $\begin{array}{c}\text { Growth of } \mathrm{K}^{+} \\
\text {uptake-deficient yeast was } \\
\text { complemented by AtCNGC11, } \\
\text { AtCNGC12 or the chimeric } \\
\text { AtCNGC11/12. Growth was } \\
\text { enhanced by } 100 \mu \mathrm{M} \\
\text { db-cAMP but not db-cGMP. }\end{array}$ & [53] \\
\hline $\begin{array}{l}\text { AtCNGC11, } \\
\text { AtCNGC12 }\end{array}$ & Yeast & $\begin{array}{c}\text { Tested } \mathrm{Ca}^{2+} \\
\text { uptake }\end{array}$ & No & $\begin{array}{l}\text { Expression of AtCNGC11, } \\
\text { AtCNGC12 or AtCNGC11/12 } \\
\text { complemented growth of } \mathrm{Ca}^{2+} \\
\text { uptake-deficient yeast. }\end{array}$ & [51] \\
\hline $\begin{array}{l}\text { AtCNGC11, } \\
\text { AtCNGC12 }\end{array}$ & Yeast & Tested $\mathrm{K}^{+}$uptake & No & $\begin{array}{l}\text { AtCNGC11/12 or AtCNGC12 } \\
\text { restored growth of } \mathrm{K}^{+} \\
\text {uptake-deficient yeast. }\end{array}$ & [81] \\
\hline $\begin{array}{l}\text { AtCNGC11, } \\
\text { AtCNGC12 }\end{array}$ & $\begin{array}{c}\text { Xenopus } \\
\text { oocytes- } \\
\text { whole cell }\end{array}$ & $\begin{array}{l}\text { Tested } \mathrm{Ca}^{2+} \\
\text { conductance }\end{array}$ & Yes & $\begin{array}{c}\text { Expression of } A t C N G C 12 \\
\text { caused a Ca }{ }^{2+} \text { conductance } \\
\text { that was not enhanced by } \\
\text { cNMPs. AtCNGC11 } \\
\text { expression did not cause a } \\
\mathrm{Ca}^{2+} \text { conductance, even with } \\
\text { cNMPs. Co-expression did } \\
\text { not affect the } \\
\text { AtCNGC12-dependent } \\
\text { conductance. }\end{array}$ & [8] \\
\hline AtCNGC14 & $\begin{array}{l}\text { Xenopus } \\
\text { oocytes- } \\
\text { whole cell }\end{array}$ & $\begin{array}{l}\text { Tested } \mathrm{Ca}^{2+} \\
\text { conductance }\end{array}$ & No & $\begin{array}{c}\text { AtCNGC14 } \mathrm{Ca}^{2+} \text { conductivity } \\
\text { was observed in the absence } \\
\text { of cNMPs. It was not tested } \\
\text { whether application of cNMPs } \\
\text { would increase } \mathrm{Ca}^{2+} \\
\text { conductance. }\end{array}$ & [57] \\
\hline AtCNGC14 & $\begin{array}{c}\text { Xenopus } \\
\text { oocytes- } \\
\text { whole cell }\end{array}$ & $\begin{array}{l}\text { Tested } \mathrm{Ca}^{2+} \\
\text { conductance }\end{array}$ & No & $\begin{array}{l}\text { AtCNGC14 inward } \mathrm{Ca}^{2+} \\
\text { currents were observed in the } \\
\text { absence of cNMPs. }\end{array}$ & [56] \\
\hline AtCNGC18 & E. coli & $\begin{array}{c}\text { Tested } \mathrm{Ca}^{2+} \\
\text { uptake }\end{array}$ & No & $\begin{array}{c}\text { Expression of } A t C N G C 18 \text { in } E \text {. } \\
\text { coli caused } \mathrm{Ca}^{2+} \\
\text { accumulation. }\end{array}$ & [62] \\
\hline AtCNGC18 & $\begin{array}{l}\text { HEK293T- } \\
\text { whole cell }\end{array}$ & $\begin{array}{l}\text { Tested } \mathrm{Ca}^{2+} \\
\text { conductance }\end{array}$ & Yes & $\begin{array}{c}\text { Application of } 100 \mu \mathrm{M} \\
\text { 8Br-cAMP or } 100 \mu \mathrm{M} \\
\text { 8Br-cGMP stimulated greater } \\
\text { AtCNGC18 } \mathrm{Ca}^{2+} \text { conductance, } \\
\text { but not } 20 \mu \mathrm{M} \text { 8Br-cNMP. Ca }{ }^{2+} \\
\text { conductance did not require } \\
\text { 8Br-cNMP application. }\end{array}$ & [5] \\
\hline
\end{tabular}


Table 2. Cont.

\begin{tabular}{|c|c|c|c|c|c|}
\hline CNGCs & System & Tested Cations & Tested with cNMPs? & Results & References \\
\hline AtCNGC18 & $\begin{array}{l}\text { Xenopus } \\
\text { oocytes- } \\
\text { whole cell }\end{array}$ & $\begin{array}{l}\text { Tested } \mathrm{Ca}^{2+} \\
\text { conductance }\end{array}$ & Yes & $\begin{array}{c}\text { In the presence of } 100 \mu \mathrm{M} \\
\mathrm{db}-\mathrm{cAMP}, \mathrm{AtCNGC18} \\
\text { conducted } \mathrm{Ca}^{2+} . \text { No data } \\
\text { were reported concerning } \\
\text { conductance in the absence of } \\
\text { db-cAMP. }\end{array}$ & [63] \\
\hline AtCNGC18 & $\begin{array}{l}\text { Pollen tube } \\
\text { protoplasts- } \\
\text { whole cell }\end{array}$ & $\begin{array}{l}\text { Tested } \mathrm{Ca}^{2+} \\
\text { conductance }\end{array}$ & Yes & $\begin{array}{c}\text { Application of } 100 \mu \mathrm{M} \\
\text { 8Br-cGMP stimulated } \\
\text { AtCNGC18-dependent } \mathrm{Ca}^{2+} \\
\text { conductance. } \mathrm{Ca}^{2+} \\
\text { conductance was not apparent } \\
\text { in the absence of } 8 \mathrm{Br}-\mathrm{cGMP} \text {. }\end{array}$ & [6] \\
\hline AtCNGC18 & $\begin{array}{l}\text { Xenopus } \\
\text { oocytes- } \\
\text { whole cell }\end{array}$ & $\begin{array}{l}\text { Tested } \mathrm{Ca}^{2+} \\
\text { conductance }\end{array}$ & No & $\begin{array}{l}\text { AtCNGC18 } \mathrm{Ca}^{2+} \text { conductivity } \\
\text { was observed in the absence } \\
\text { of cNMPs. Co-expression of } \\
\text { AtCNGC18 with AtCNGC7 or } \\
\text { AtCNGC8 eliminated } \mathrm{Ca}^{2+} \\
\text { conductivity. }\end{array}$ & [46] \\
\hline AtCNGC19 & $\begin{array}{l}\text { Xenopus } \\
\text { oocytes- } \\
\text { whole cell }\end{array}$ & $\begin{array}{l}\text { Tested } \mathrm{Ca}^{2+}, \mathrm{Na}^{+} \\
\text {and } \mathrm{K}^{+} \\
\text {conductance }\end{array}$ & Yes & $\begin{array}{c}\text { In the presence of } 300 \mu \mathrm{M} \\
\mathrm{db}-\mathrm{cAMP} \text {, AtCNGC19 elicited } \\
\mathrm{Ca}^{2+} \text { inward currents but not } \\
\mathrm{K}^{+} \text {and } \mathrm{Na}^{+} \text {currents. }\end{array}$ & [66] \\
\hline $\begin{array}{l}\text { AtCNGC19, } \\
\text { AtCNGC20 }\end{array}$ & $\begin{array}{l}\text { Xenopus } \\
\text { oocytes- } \\
\text { whole cell }\end{array}$ & $\begin{array}{l}\text { Tested } \mathrm{Ca}^{2+} \\
\text { conductance }\end{array}$ & No & $\begin{array}{l}\text { AtCNGC19 and AtCNGC20 } \\
\text { conductivity was observed in } \\
\text { the absence of cNMPs. It was } \\
\text { not tested whether } \\
\text { application of cNMPs would } \\
\text { increase } \mathrm{Ca}^{2+} \text { conductance. } \\
\text { Co-expression of } A t C N G C 19 \\
\text { and AtCNGC20 increased } \\
\text { conductance compared to } \\
\text { independently expressed } \\
\text { AtCNGC19 and AtCNGC20. }\end{array}$ & [67] \\
\hline $\mathrm{PpCNGCb}$ & $\begin{array}{l}\text { Moss } \\
\text { protoplasts } \\
\text { cell attached }\end{array}$ & $\begin{array}{c}\text { Tested } \mathrm{Ba}^{2+} \\
\text { conductance (as } \\
\text { a proxy for } \mathrm{Ca}^{2+} \text { ) }\end{array}$ & No & $\begin{array}{l}\mathrm{Ba}^{2+} \text { conductivity did not } \\
\text { require application of cNMPs, } \\
\text { but it is possible that there } \\
\text { were endogenous cNMPs. } \\
\mathrm{Ba}^{2+} \text { conductivity was altered } \\
\text { in Ppcngcb mutants. }\end{array}$ & [28] \\
\hline HvCNGC2-3 & $\begin{array}{l}\text { Xenopus } \\
\text { oocytes- } \\
\text { whole cell }\end{array}$ & $\begin{array}{c}\text { Tested } \mathrm{K}^{+} \text {and } \\
\mathrm{Na}^{+} \text {conductance }\end{array}$ & Yes & 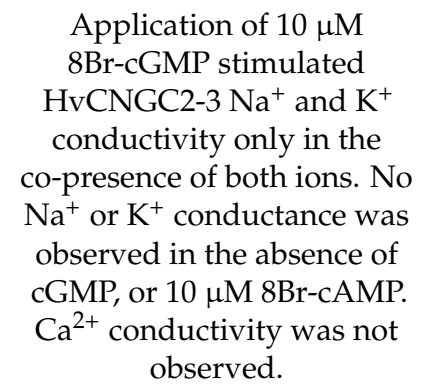 & [7] \\
\hline
\end{tabular}


Table 2. Cont.

\begin{tabular}{|c|c|c|c|c|c|}
\hline CNGCs & System & Tested Cations & Tested with cNMPs? & Results & References \\
\hline OsCNGC9 & $\begin{array}{l}\text { HEK293- } \\
\text { whole cell }\end{array}$ & $\begin{array}{l}\text { Tested } \mathrm{Ca}^{2+} \text { and } \\
\mathrm{K}^{+} \text {conductance }\end{array}$ & No & $\begin{array}{c}\text { OsCNGC9 } \mathrm{Ca}^{2+} \text { conductivity } \\
\text { was observed in the absence } \\
\text { of cNMPs. Comparatively } \\
\text { little } \mathrm{K}^{+} \text {conductivity was } \\
\text { observed. It was not tested } \\
\text { whether application of cNMPs } \\
\text { would increase } \mathrm{Ca}^{2+} \text { or } \mathrm{K}^{+} \\
\text {conductance. }\end{array}$ & [89] \\
\hline OsCNGC13 & $\begin{array}{l}\text { HEK293- } \\
\text { whole cell }\end{array}$ & $\begin{array}{l}\text { Tested } \mathrm{Ca}^{2+} \text { and } \\
\mathrm{K}^{+} \text {conductance }\end{array}$ & No & $\begin{array}{c}\text { OsCNGC13 mediated } \mathrm{Ca}^{2+} \\
\text { inward currents but not } \mathrm{K}^{+} \\
\text {currents. It was not tested } \\
\text { whether application of cNMPs } \\
\text { would increase } \mathrm{Ca}^{2+} \\
\text { conductance. }\end{array}$ & [90] \\
\hline MtCNGC15 & $\begin{array}{c}\text { Xenopus } \\
\text { oocytes- } \\
\text { whole cell }\end{array}$ & $\begin{array}{c}\text { Tested } \mathrm{Ba}^{2+} \text { and } \\
\mathrm{Ca}^{2+} \\
\text { conductance }\end{array}$ & No & $\begin{array}{c}\text { MtCNGC15 } \mathrm{Ca}^{2+} \text { conductivity } \\
\text { did not require application of } \\
\text { cNMPs. It was not tested } \\
\text { whether application of cNMPs } \\
\text { would increase } \mathrm{Ca}^{2+} \\
\text { conductance. }\end{array}$ & [77] \\
\hline
\end{tabular}

\section{CNGCs Are Extensively Regulated-Formation of CNGC Complexes Generates Further Regulatory and Functional Complexity}

Here we summarise the current understanding of CNGC structure and regulation, discussing how the formation of CNGC complexes may further affect CNGC regulation and function. The breakthrough studies on plant CNGCs used primary structures of potassium channels and animal CNG subunits to assign domains [91-93]. The overall model CNGC subunit has six transmembrane domains (S1-S6; Figure 2) with a pore region (P loop) between S5 and S6 that permits ion transport [13]. Animal and bacterial cation channel subunits that contain a single P loop form tetramers; this includes animal CNGs, with clear evidence from cryo-electron microscopy showing tetramer formation in a lipid environment [94]. Triplet amino acid residues in the $\mathrm{P}$ loop that could act as selectivity filters (AGN, AND, GNL, GQG, GQN, GQS) vary between the Arabidopsis CNGCs [95], with AND or GQs thought to confer some level of $\mathrm{Ca}^{2+}$ selectivity $[4-6,30,35,45,78]$. Recent analysis of AtCNGCs has revealed the presence of a diacidic motif for $\mathrm{Mg}^{2+}$ binding close to the pore region in the cytosolic CT in all but AtCNGC2. It has been proposed that this could account for channel blocking by cytosolic $\mathrm{Mg}^{2+}$; the consequences for signalling and nutrition have yet to be explored [96]. The CT contains a cyclic nucleotide-binding domain (CNBD) which is believed to be formed of four $\alpha$-helices $(\alpha \mathrm{A}, \alpha \mathrm{P}, \alpha \mathrm{B}, \alpha \mathrm{C})$ and eight $\beta$-sheets ( $\beta 1-\beta 8$ ). Overlapping with the $C$-terminal side of the CNBD is a calmodulin (CaM)-binding domain (CaMBD) $[97,98]$. A CaM-binding IQ (isoleucine-glutamine) motif is also present $[18,21,23,82]$ and the $C T$ can contain multiple phosphorylation sites $[29,67,89]$. The CT of AtCNGC8 appears necessary and sufficient for interactions between AtCNGC8 and AtCNGC18 subunits in Xenopus oocytes [46]. There is also a short, cytosolic N-terminal domain (NT) that is predicted to harbour CNGC-CNGC interaction domains [99] and may contain phosphorylation sites [29,67]. The NT of AtCNGC12 contains a CaMBD [50]. The AtCNGC19 and AtCNGC20 NTs are predicted to have cysteine residues that could form an $\mathrm{Fe} / \mathrm{Cu}$-binding site to act as a Fenton catalyst in the production of hydroxyl radicals regulate plant $\mathrm{Ca}^{2+}$ channel activity in growth and stress responses $[99,100]$. 


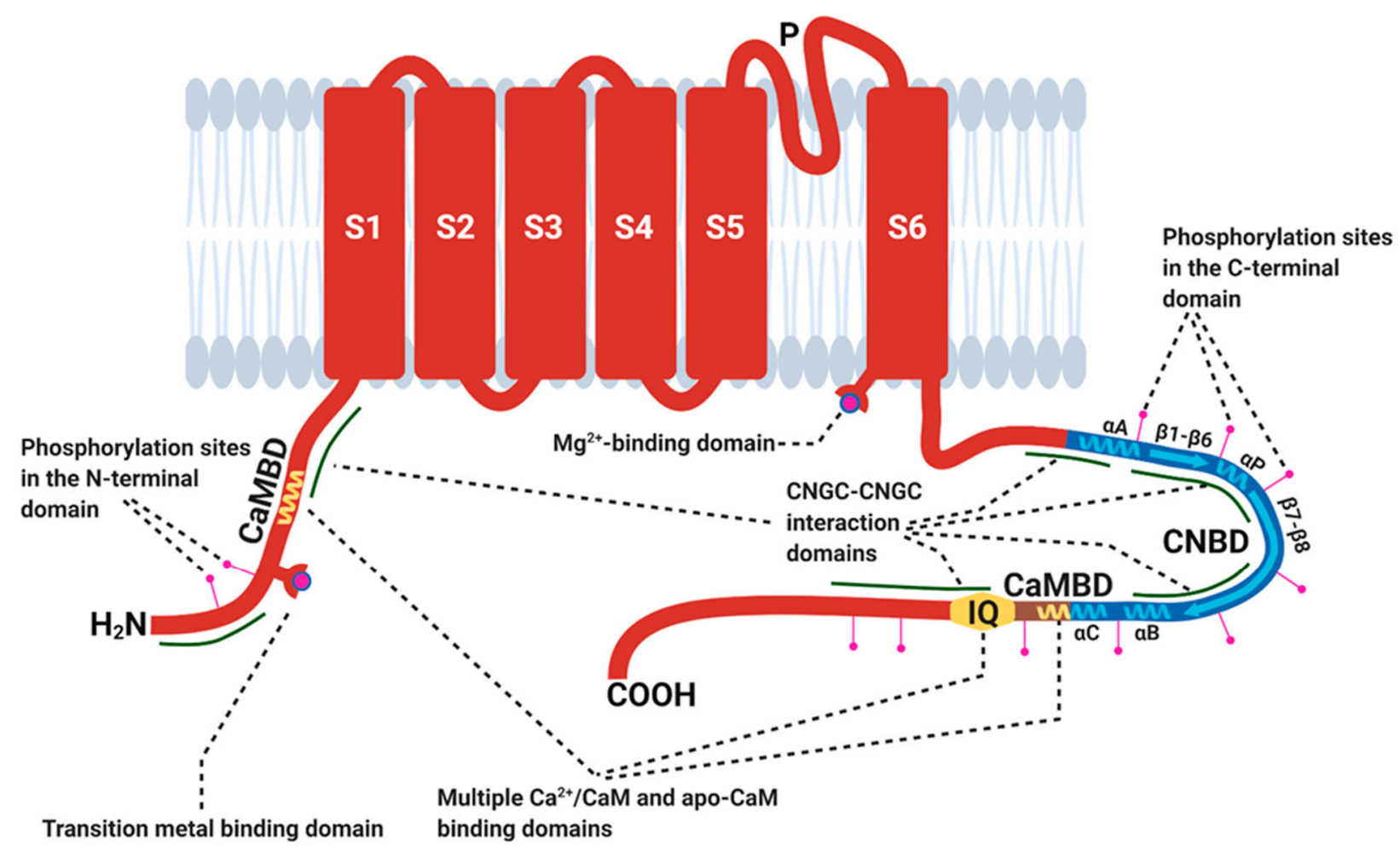

Figure 2. Cyclic nucleotide-gated channel (CNGC) subunit structure. Plant CNGCs consist of six transmembrane domains (S1-S6) with a pore region between S5 and S6. Both $\mathrm{N}$ - and C-terminal domains are cytosolic. In the C-terminal domain is a cyclic nucleotide-binding domain (CNBD), which is believed to be formed of four $\alpha$-helices $(\alpha \mathrm{A}, \alpha \mathrm{P}, \alpha \mathrm{B}, \alpha \mathrm{C})$ and eight $\beta$-sheets $(\beta 1-\beta 8)$. Overlapping with the C-terminal side of the CNBD is a calmodulin (CaM)-binding domain (CaMBD). CNGCs also contain a CaM-binding IQ (isoleucine-glutamine) motif. From the data presented by Pan et al. 2019 [46] and Chiasson et al. 2017 [99], the N- and C-terminal regions are predicted to contain CNGC-CNGC interaction domains. Lemtiri-Chlieh et al. 2020 [96] predict the presence of a $\mathrm{Mg}^{2+}$-binding domain downstream of the CNGC pore. Demidchik et al. 2014 [99] predict a transition metal-binding domain in the N-terminus of AtCNGC19 and AtCNGC20. CNGC phosphorylation is likely for AtCNGC4 [29], AtCNGC18 [63], AtCNGC19 [67], AtCNGC20 [67] and OsCNGC9 [89]. An N-terminal CaMBD has been identified in AtCNGC12 [50]. Not all plant CNGCs contain all the structures displayed on this image. Structure was adapted from Chin et al. 2009 [12] and Kaplan et al. 2007 [101]. Figure created with BioRender.com.

\subsection{CNGCs Are Regulated by cNMPs That May Be Generated by Soluble or Membrane Proteins}

cAMP and cGMP are secondary messengers which are synthesised by adenylyl cyclases (ACs) and guanylyl cyclases (GCs), respectively. cNMP gating of CNGCs is well documented and is summarised in Table 2, but historically the physiological importance of cNMPs has been controversial [11,102-104]. cNMP levels in plants are significantly lower than in animals and, until the development of more sensitive assays, it was doubted whether cNMPs were present at all [102]. To date, cAMP has been implicated in seed germination and cell cycle progression, pollen tube growth and orientation, stomatal kinetics, photosynthesis and photorespiration, abiotic stress responses (heat and chill stress, salinity, drought, aluminium, nutrient deficiency), wounding and immunity [11]. A range of soluble and membrane proteins has now been identified with potential AC or GC activity, in their cytosolic domains for the membrane proteins. For ACs, these include the $\mathrm{K}^{+}$ uptake transporters AtKUP5 and AtKUP7 ( $\mathrm{K}^{+}$uptake permease) $[105,106]$, and AtLRRAC1 (leucine-rich repeat adenylyl cyclase1) [107]. For GCs, AtGC1 (guanylyl cyclase1) [108], AtNOGC1 (nitric oxide-dependent guanylate cyclase1) [109], AtPSKR1 (phytosulfokine receptor1) [110], AtPepR1 (plant elicitor peptide receptor1) [3], AtBRI1 (brassinosteroid insenstive1) [111] and AtWAKL10 (wall-associated kinase (WAK)-like10) [112] have all been identified and studied in vitro. Two homologues of AtPepR1 have now been identified 
in tomato (Solanum lycopersicum L.; SlGC17, SlGC18) and have been reported to have GC activity that is required for $\left[\mathrm{Ca}^{2+}\right]_{\text {cyt }}$ increase in response to flg22, chitin and AtPep1 [113]. Analysis of recombinant protein activity suggests that AC and GC activity in plants is typically much lower than in animal counterparts, with pmol or fmol cNMP $\mu \mathrm{g}^{-1}$ protein min $^{-1}$ values reported [3,107-109,111]. However, the membrane-bound AtWAKL10 and AtPSKR1 have a $V_{\max }$ of approximately $2 \mu \mathrm{mol} \mathrm{mg} \mathrm{min}^{-1}$ [110,112]. It is possible that, in planta, CNGC cNMP sensitivity is increased by CaM, phosphorylation, or formation of heterotetrameric complexes, and so lower cNMP concentrations are required than those used in heterologous expression systems or native membranes in transport studies.

It may be that the low activity of plant ACs and GCs is central to signal specificity. If the domains were in close proximity to specific CNGCs, generating cNMP concentrations sufficient to activate those channels [3], then crosstalk between separate CNGC-dependent signalling pathways could be eliminated-only CNGCs co-localising with the AC or GC would be activated. How close is close enough? At the plasma membrane, both AtCNGC17 and AtPSKR1 have been found to interact with AtBAK1 (BRI-associated receptor kinase1) and although AtCNGC17 does not interact with the PSKR1 receptor that generates cGMP, this channel is essential for phytosulfokine/PSKR1-dependent protoplast expansion involving the $\mathrm{H}^{+}$-ATPases AtAHA1 and AtAHA2 and is thought to form part of this multi-protein complex [60]. Increased $\left[\mathrm{Ca}^{2+}\right]_{\text {cyt }}$ promotes PSKR1's GC activity but inhibits its kinase activity [114], raising the possibility that AtCNGC17-mediated $\left[\mathrm{Ca}^{2+}\right]_{\mathrm{cyt}}$ elevation not only generates a positive feedback loop for the cGMP pathway but could also curtail any phosphorylation-dependent pathway to ensure signalling specificity. A positive feedback loop may also explain the jasmonic acid-induced rise in cAMP in leaf epidermal cells that requires AtCNGC2 [35]. It is possible that AtCNGC2-mediated $\mathrm{Ca}^{2+}$ influx activates ACs either directly or via intermediates such as calcium-dependent protein kinases and CaMs.

The recent finding that Arabidopsis root hair $\mathrm{K}^{+}$influx precedes increased growth rate and can cause $\left[\mathrm{Ca}^{2+}\right]_{\mathrm{cyt}}$ increase [115] begs the question of whether AC activity of AtKUP5 and AtKUP7 is involved. Both these $\mathrm{K}^{+}$transporters are expressed in root hairs [116], and AtKUP7 is in the plasma membrane [117]. When expressed in yeast, AtKUP5-mediated $\mathrm{K}^{+}$ influx causes cAMP accumulation [106]. This suggests a model in which KUP-mediated $\mathrm{K}^{+}$influx to the root hair causes cAMP increase to activate the CNGCs (AtCNGC5,6,9,14) implicated in $\mathrm{Ca}^{2+}$ influx and polar growth [43,44,55-57]. As AtCNGC5, AtCNGC6 and AtCNGC9 appear to transport $\mathrm{Ca}^{2+}$ rather than monovalent cations $[4,6,43]$ (Table 2), it seems likely these subunits are relevant to $\mathrm{Ca}^{2+}$ signalling. The spatial localisation of the KUPs relative to the CNGCs is worthy of attention. Activation of CNGCs with strong $\mathrm{K}^{+}$ permeation could conceivably contribute to $\mathrm{K}^{+}$uptake in root hairs and other cells, indeed AtCNGC3 and AtCNGC10 are held to be important for root $\mathrm{K}^{+}$acquisition $[48,101]$.

Salt stress causes cGMP accumulation within seconds in Arabidopsis seedlings [118] and CNGCs have been proposed to be part of the salt-induced $\left[\mathrm{Ca}^{2+}\right]_{\text {cyt }}$ signalling response [119]. In Arabidopsis, cNMPs can restrict $\mathrm{Na}^{+}$influx rather than promote it, ostensibly by reducing the open probability of root plasma membrane $\mathrm{Na}^{+}$-permeable channels (an effect observed in approximately half of the patch clamp trials) [120]. This would imply negative regulation by cNMPs of a putative CNGC channel. Notably, AtCNGC3 and AtCNGC10 appear to contribute to $\mathrm{Na}^{+}$uptake [40,49]. Patch clamp electrophysiological analysis of Arabidopsis root epidermal plasma membrane has also revealed a $\mathrm{Na}^{+}$influx channel that could not discriminate against $\mathrm{K}^{+}$[121] (a "non-selective" cation channel [122]) and this was proposed to be $\mathrm{Ca}^{2+}$ permeable in a later study [123]. It is not known whether the channel is regulated by cNMPs and could account for the negative effects of cNMPs on $\mathrm{Na}^{+}$influx reported by Maathuis and Sanders, 2001 [120]. The roles of cNMPs and CNGCs in salt stress urgently require further elucidation. Understanding which CNGC subunits and potential heteromeric complexes are expressed in different root cells (which vary in their salt-induced $\left[\mathrm{Ca}^{2+}\right]_{\text {cyt }}$ response [124]), what their functional permeability is to $\mathrm{Na}^{+}$ and $\mathrm{Ca}^{2+}$ and how they are regulated by cNMPs is likely to be of great importance. 
4.2. CNGCs Are Positively and Negatively Regulated by CaM, Potentially Affording $\mathrm{Ca}^{2+}$ Sensing and Feedback

Calmodulins are $\mathrm{Ca}^{2+}$-binding proteins with a major role in $\mathrm{Ca}^{2+}$ signal transduction in plants $[10,29,46]$. In former models of CNGC activity, CaM was believed to have an exclusively inhibitory effect where the $\mathrm{Ca}^{2+}$-bound form of $\mathrm{CaM}\left(\mathrm{Ca}^{2+} / \mathrm{CaM}\right)$ inhibited CNGCs by perturbing cNMP gating, competing for a binding site internal to the CNBD [98]. Subsequently, multiple CaMBDs have been identified, with structural divergence amongst CNGC isoforms. For example, AtCNGC12 contains an N-terminal CaMBD which interacts with $\mathrm{Ca}^{2+} / \mathrm{CaM}$ and could result in channel closure [50]. In addition, an IQ domain has been identified which is C-terminal to the CNBD and conserved in the majority of plant CNGCs $[18,21,23,85]$. Yeast 2-hybrid assays suggest that interactions between CNGC $\mathrm{CTs}$ and $\mathrm{CaM}$ isoforms are specific, with the IQ domain contributing to many of these interactions [33]. Indeed, CaM isoform-specific effects are now being documented [10], for example root hair AtCNGC14 is negatively regulated by AtCaM7 binding to its CT but not by AtCaM2 [56]. Additionally, AtCNGC6 is inhibited by AtCaM2,3,5,7 at the IQ domain in heat shock signalling but not by AtCaM1,4 or 6 [125].

It has been proposed that apo-CaM (CaM without $\mathrm{Ca}^{2+}$ ligands) constitutively binds to the IQ domain in a $\mathrm{Ca}^{2+}$-independent manner to act as a $\mathrm{Ca}^{2+}$ sensor $[10,33,50]$. The model arising from studies on AtCNGC12 has the channel's opening causing local $\mathrm{Ca}^{2+}$ elevation, hence permitting $\mathrm{Ca}^{2+}$ binding to apo-CaM [50]. That initial channel opening could be triggered by membrane hyperpolarisation because when expressed in Xenopus, AtCNGC12 presents as a hyperpolarisation-activated $\mathrm{Ca}^{2+}$ channel that does not require cNMPs [8]. $\mathrm{Ca}^{2+}-\mathrm{CaM}$ interaction at the IQ domains of adjacent subunits and $\mathrm{Ca}^{2+}-\mathrm{CaM}$ recruitment to CaMBDs could modulate channel activity [50]. Evidence from Xenopus expression points to AtCaM1 as an activating ligand [8]. As $\mathrm{Ca}^{2+}$ increases, $\mathrm{Ca}^{2+}-\mathrm{CaM}$ binding to the NT CaMBD effects channel closure [10,50]. Much, therefore, depends on which CaM isoforms are locally available and their affinities for $\mathrm{Ca}^{2+}$ and the CaMBDs. A further model built on AtCNGC8/18 activity in Xenopus coupled with analysis of CT binding has apo-CaM2 binding to the IQ domains to counter the inhibitory effect of AtCNGC8 and so promote channel opening. As $\mathrm{Ca}^{2+}$ increases as a consequence, $\mathrm{Ca}^{2+}-\mathrm{CaM} 2$ forms but then dissociates to promote channel closure [46]. Expressing AtCNGC 8 and AtCNGC18 with AtCaM2 in HEK293 cells leads to $\left[\mathrm{Ca}^{2+}\right]_{\text {cyt }}$ oscillations [46], which has implications for pollen tube apical $\left[\mathrm{Ca}^{2+}\right]_{\text {cyt }}$ oscillations during growth. It remains to be seen how cNMPs fit into this regulatory complex, given activation of AtCNGC18 by cGMP in native pollen plasma membrane [6] and by cNMPs in heterologous expression $[5,63]$.

CaM regulation of CNGCs may be important in immune signalling. BIK1 is a receptorlike cytoplasmic kinase which acts downstream of FLS2 (Flagellin Sensitive2) [126], an LRR receptor-like kinase which binds to the bacterial flg22 peptide and is required for $\left[\mathrm{Ca}^{2+}\right]_{\mathrm{cyt}}$ elevation [127]. Although at the whole-plant level (which may lack sufficient resolution) AtCNGC2 was reported to have no involvement in flg22-induced $\left[\mathrm{Ca}^{2+}\right]_{\text {cyt }}$ increase [128], a genetic analysis of $A t C N G C 2$ and AtCNGC4 concluded that both genes act in the flg22 pathway [27]. At the leaf disc level, use of loss-of-function mutants indicated that both AtCNGC2 and AtCNGC4 are involved in flg22-induced $\left[\mathrm{Ca}^{2+}\right]_{\mathrm{cyt}}$ increase, given a permissive apoplastic $\mathrm{Ca}^{2+}$ level [29]. Similarly, patch clamping of mesophyll protoplasts showed that both were needed for flg22-induced plasma membrane $\mathrm{Ca}^{2+}$ influx currents [29]. It should be noted, however, that flg22-induced plasma membrane depolarisation of individual mesophyll cells (which involves $\mathrm{Ca}^{2+}$ influx) was found to be independent of AtCNGC2 [129]. Following the results of heterologous co-expression in Xenopus oocytes [29], it is likely the subunits form a AtCNGC2/4 complex. Although single expression of either AtCNGC2 or AtCNGC4 in Xenopus oocytes failed to cause channel activity, their co-expression produced a hyperpolarisation-activated $\mathrm{Ca}^{2+}$-permeable channel that did not require cNMPs [29]. This channel activity could be supressed by the co-expression of AtCAM7 and this suppression could be overcome by the additional expression of AtBIK1 [29]. It was subsequently found that application of flg22 induces AtBIK1-mediated phosphorylation of the AtCNGC4- 
CT in planta, which is believed to overcome AtCaM7-mediated repression [9,29]. More recently, a split luciferase complementation assay using nano-luciferase suggests that the $\mathrm{CT}$ and NT of homomeric AtCNGC2 and AtCNGC4 may move apart when challenged with flg22 in planta [130]. It may be postulated that this change is linked to the disassembly of homomeric complexes and the formation of heterotetrameric complexes. Facultative complex formation may contribute to the ability for CNGC subunits to carry out multiple signalling roles.

Again, it remains to be seen what role, if any, cNMPs play in this pathway. Electrophysiological analyses support cNMP activation of AtCNGC2 either in native membrane or when heterologously expressed (Table 2; [30,38]) and with an apparent ability to discriminate between cAMP and cGMP in guard cells $[4,30]$. As flg22 can induce guard cell $\left[\mathrm{Ca}^{2+}\right]_{\text {cyt }}$ oscillations [131] and as CNGCs could be involved in $\left[\mathrm{Ca}^{2+}\right]_{\text {cyt }}$ oscillations [46], further consideration of guard cell AtCNGC2 in this immune pathway is warranted. The recent discovery that BIK1 phosphorylates the guard cell plasma membrane $\mathrm{Ca}^{2+}$ channel AtOSCA1.3 (hyperOsmolality-induced $\left[\mathrm{Ca}^{2+}\right]$ i increase 1.3) as part of the stomatal flg22 response [132] still leaves room for other $\mathrm{Ca}^{2+}$ influx pathways. Moving away from Arabidopsis, SlCNGC1 and SICNGC14 are required for the tomato flg22-induced $\left[\mathrm{Ca}^{2+}\right]_{\mathrm{cyt}}$ increase but it is not yet clear whether these subunits can form a complex [133]. Overall, the study of CNGC regulation by CaM is complicated by the abundance of CaM and CNGC isoforms and the multitude of CaMBDs. It is likely that this complexity contributes to the specificity of signal transduction by CNGCs.

\subsection{CNGCs Are Regulated by Phosphorylation}

CNGC phosphorylation is emerging as an important regulator of activity. AtCNGC4 contains nine phosphorylation sites within and around the CT CNBD and, as described in Section 4.2, phosphorylation by BIK1 relieves CaM7-mediated inhibition of the putative AtCNGC2/4 complex in flg22 signalling [29]. Similarly, the rice receptor-like cytoplasmic kinase OsRLCK185 (receptor-like cytoplasmic kinase 185) is responsible for activation of OsCNGC9 by phosphorylation, triggering defence responses [89]. AtCNGC19 and AtCNGC20 have also been found to play a role in plant defence downstream of BAK1/SERK4 (somatic embryogenesis receptor kinase 4). However, in this case, the authors proposed that the signalling cascade progressed through BAK1-mediated phosphorylation of the AtCNGC20-CT, leading to proteasome-dependent degradation, as opposed to phosphorylation-mediated channel activation [67]. Mutation of $\mathrm{Thr}^{560} / \mathrm{Ser}^{617} / \mathrm{Ser}^{618} / \mathrm{Thr}^{619}$ in the AtCNGC20-CT reduced BAK1-mediated phosphorylation, and additional phosphorylation sites were predicted in the C- and N-terminals [67]. It was not reported, however, whether AtCNGC20-CT phosphorylation might also be an activating signal, which may subsequently be followed by signal termination via protein degradation. It is possible, therefore, that AtCNGC20-CT phosphorylation may overcome CaM-mediated inhibition, as found with AtCNGC2/4 in the flg22 pathway. It is also possible that AtCNGC2/4 phosphorylation may promote proteasome-mediated degradation and, as such, CNGC phosphorylation may have dual function in planta.

The role of CNGC phosphorylation is not restricted to defence signalling. Calciumdependent protein kinase 32 (CPK32) appears to interact with AtCNGC18 in planta, increases the conductance of AtCNGC18 homotetramers when co-expressed in Xenopus oocytes and, following overexpression in pollen tubes, leads to increased apical $\left[\mathrm{Ca}^{2+}\right]_{\text {cyt }}[63]$. Following the identification of AtCNGC8/18 heterotetramers, it would be interesting to test how CPK32 affects AtCNGC8/18 activity. In silico analysis also predicts numerous phosphorylation sites in CNGCs from N. tabacum [18] and Brassica oleracea [22], suggesting that kinase/phosphatase regulation of CNGC activity is widespread.

\subsection{CNGC Complexes Generate Further Functional and Regulatory Complexity}

As discussed, the functional characteristics of heterologously expressed AtCNGC8/18 and the putative AtCNGC2/4 complex can be different from the homotetrameric channels 
of their constituent subunits, including changes to cNMP gating $[29,46]$. It also appears that CNGC complexes display changes in ion selectivity. AtCNGC2 can conduct $\mathrm{K}^{+}, \mathrm{Cs}^{+}$, $\mathrm{Rb}^{+}$and $\mathrm{Li}^{+}$but has a very low permeability to $\mathrm{Na}^{+}$which correlates with a change in pore region amino acid sequence, from GQN to AND [85]. It would appear, therefore, that AtCNGC2 has a role in mineral nutrition where $\mathrm{Na}^{+}$permeability is particularly deleterious. AtCNGC2 has been implicated in uptake of $\mathrm{Ca}^{2+}$ into leaves [38] and it may be that $\mathrm{Na}^{+}$exclusion is important in this function. Unlike AtCNGC2, AtCNGC4 appears to be permeable to both $\mathrm{K}^{+}$and $\mathrm{Na}^{+}$[41]. However, co-expression of AtCNGC2 and AtCNGC4 forms channels which are permeable to $\mathrm{K}^{+}$but impermeable to $\mathrm{Na}^{+}$[29]. This supports the hypothesis that AtCNGC2 and AtCNGC4 do form complexes and suggests that these complexes have unique functional characteristics, including permeability. It is important for future studies of CNGC permeability, therefore, to consider whether the CNGCs being tested exist as complexes in planta. For example, Zhang et al. reported that expression of AtCNGC14 in Xenopus oocytes produced channels which were permeable to $\mathrm{Mg}^{2+}$ but effectively impermeable to $\mathrm{K}^{+}, \mathrm{Na}^{+}$and $\mathrm{Ba}^{2+}$, under the conditions tested [57]. It should be investigated whether AtCNGC complexes containing AtCNGC14 also display selectivity against these ions.

CNGC complexes will also make allosteric regulation of CNGC activity more intricate. For example, since CNGC complexes will be composed of multiple CNGC isoforms, with non-identical CTs and NTs (Figure 3A), the CNGC complex may interact with new combinations of allosteric regulators, including different $\mathrm{CaM}$ isoforms or different protein kinases. Each combination is likely to be unique to each CNGC complex and could result in unique feedback loops. Consequently, CNGC complexes may produce characteristic $\mathrm{Ca}^{2+}$ signatures which would enable CNGC complexes, even those that share a CNGC subunit, to participate in different signalling pathways. In addition, it is possible that the selectivity of some CNGCs, such as HvCNGC2-3 [7], for one of cAMP or cGMP may lead to specificity in heterotetrameric complexes.

An additional consideration is how CNGC subunits may compete for interactions with other CNGC isoforms. For example, it is apparent that AtCNGC7 and AtCNGC8 preferentially interact with AtCNGC18 in Xenopus to form AtCNGC7/18 or AtCNGC8/18 heterotetrameric complexes, instead of forming homotetrameric complexes [46]. It is also likely that AtCNGC2 and AtCNGC4 also preferentially interact to form AtCNGC2/4 heterotetramers, as opposed to homotetramers. Therefore, in a situation where two or more possible CNGC complexes may be formed, it is likely that particular CNGC complexes will form preferentially over others. Understanding these interaction dynamics may be key to understanding CNGC activity. The brush mutation in Lotus japonicus is an exemplar of how small changes in CNGC structure can significantly alter CNGC complex function in planta [99]. BRUSH is an LjCNGC homologous to AtCNGC19 and AtCNGC20 [99]. The brush mutation is found within the CNGC N-terminus and leads to a quantitative gain-offunction phenotype associated with the constitutive, voltage-dependent $\mathrm{Ca}^{2+}$ permeability of the brush homotetramer [99]. Competition between alternative CNGC subunits is believed to restrict formation of this homotetramer except in those plants strongly expressing brush [99].

There also remain a number of avenues which have remained unexplored in the study of plant CNGC complexes. For example, it remains unknown whether plant CNGCs form complexes with three or four different subunits (Figure 3B). Furthermore, whilst it has been assumed in models of CNGC complexes that the stoichiometry of CNGC subunits is $2: 2[10,67]$, there is only evidence supporting that assumption in the case of AtCNGC8/18 and AtCNGC7/18 [46]. It is also possible that CNGC function may be altered by the order in which CNGC subunits are ordered around the channel pore. In their analysis of animal CNG channels, Liu et al. discovered that the order of CNG subunits could alter channel conductance by up to 50\% [69]. Two CNGC complexes, therefore, whilst having the same stoichiometry of CNGC subunits, may display different functional characteristics. Perhaps the formation of CNGC complexes, and the order of CNGC subunits, in planta is influenced 
by allosteric regulators such as $\mathrm{CaM}$, which may promote stronger interactions between different CNGC subunits, and help dictate their order. Alternatively, it is possible that where the order of CNGC subunits differs, the conductance, ion selectivity and interactions with allosteric regulators are all altered, leading to divergent functional outcomes between otherwise similar CNGC complexes.

A

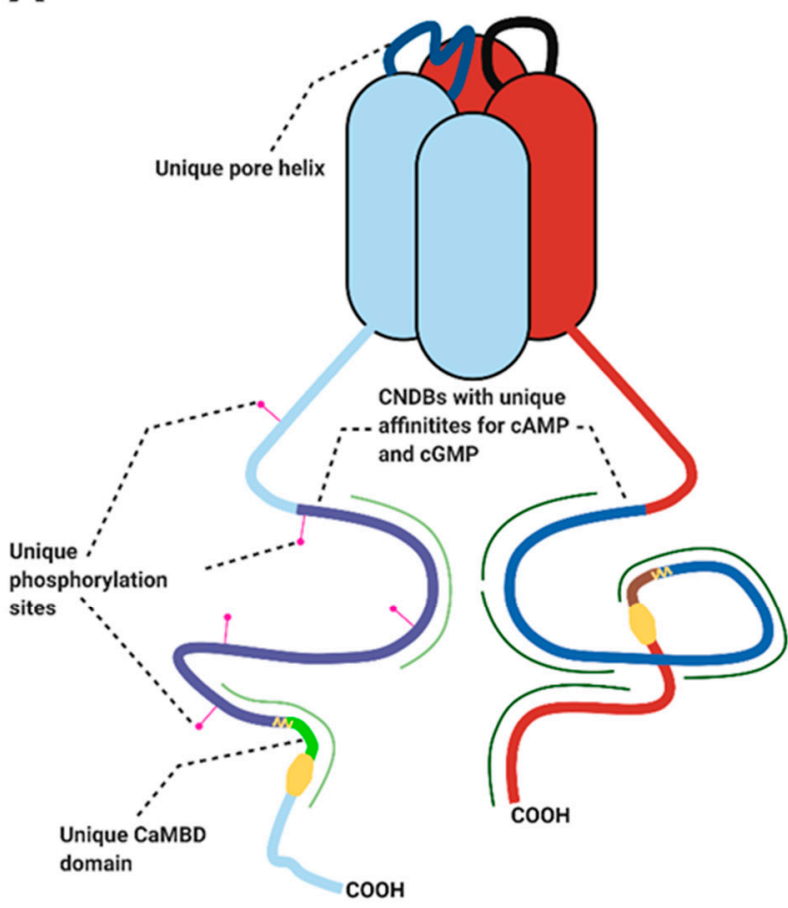

B

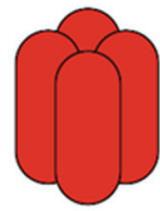

Homotetramer

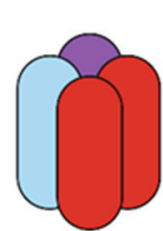

2:1:1

Heterotetramer

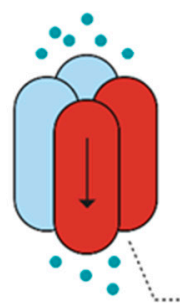

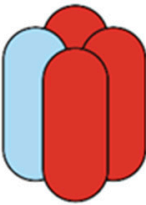

3:1

Heterotetramer

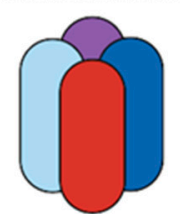

$1: 1: 1: 1$ Heterotetramer

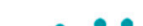

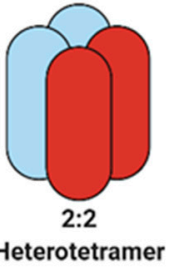

Heterotetramer

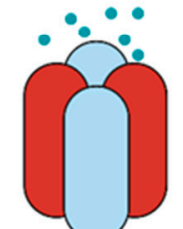

Alternative

arrangements of

CNGC subunits may

alter channel

function

Figure 3. (A) Heterotetrameric complexes possess non-identical C-terminal domains. Differences in the cyclic nucleotide binding domain may determine specificity for cGMP or cAMP. Different CaM isoforms show preferential binding with different CNGC C-terminal domains [8,33], so heterotetrameric complexes may show combinatorial control by different CaM isoforms. Different CNGC C-terminal domains will be phosphorylation targets of different protein kinases, providing a mechanism for signal integration. The combination of different pore helixes may lead to changes in CNGC ion selectivity. For simplicity not all pore helices or cytosolic domains are shown. (B) Multiple different arrangements of CNGC subunits could be possible. CNGC activity may be regulated by both the different isoforms involved and their arrangement. Figure created with BioRender.com.

In silico analysis of CNGC sequences have identified several uncharacterised motifs which may further enhance CNGC regulation and function. Nawaz et al. identified three uncharacterised NtabCNGC motifs that are approximately 50 amino acids long [18], as well as a raffinose synthase motif in all 35 NtabCNGCs [18]. Furthermore, three uncharacterised motifs in Brassica rapa CNGCs are found in a number of closely related BraCNGCs, suggesting that these BraCNGCs have additional functionality [23]. It is possible that some of these uncharacterised motifs contribute to CNGC subunit interactions and, if heterotetrameric complexes were confirmed to be widespread amongst CNGCs, these complexes would significantly increase the complexity of CNGC regulation.

\section{Could CNGCLs Modulate Complex Formation?}

Angiosperm evolution has seen the loss of several types of $\mathrm{Ca}^{2+}$ channel that are still found in animals and an apparent overall reduction in diversity of $\mathrm{Ca}^{2+}$ influx mechanisms compared to animals [134]. This suggests a greater reliance on the channels that were retained over evolution such as CNGCs. By comparison, many plant species harbour more cyclic nucleotide-gated channel subunits than animals. Vertebrates (including mammals) 
and invertebrates have only six CNGs $[94,98,135]$. Subunits of the hyperpolarisation activated cyclic nucleotide-gated (HCN) cation channels (that operate in cardiac cells) are also present in low numbers (three in invertebrates, four in mammals and four to six in other vertebrates) [136]. An ability to form diverse CNGC complexes from a greater number of subunits could compensate for the reduction in diversity of $\mathrm{Ca}^{2+}$ influx mechanism evident in plant genomes and fit each cell to respond appropriately to the diverse and coincident stimuli experienced during their sessile lives. Additionally, a range of homomeric or heteromeric CNGC channels could permit function beyond $\mathrm{Ca}^{2+}$ signalling and help explain the role of CNGCs in mineral nutrition. Truncated, CNGC-like (CNGCL) proteins may also provide a further layer of regulation by modulating CNGC complex formation (Figure 4). Pan et al. demonstrated that the AtCNGC8 CT inhibits AtCNGC18 activity [46]. This is likely due to the formation of AtCNGC $8 / 18$ heterotetramer-like interactions which prevent formation of the AtCNGC18 homotetramer. In principle, therefore, any CNGC CT could disrupt CNGC-CNGC interactions. Likewise, since the data presented by Chiasson et al. suggest that the CNGC NT also contains CNGC-CNGC interaction domains [137], it is possible that any CNGC NT could also disrupt CNGC-CNGC interactions. Genomewide analysis of $C N G C$ sequences has identified a number of truncated CNGC genes in $B$. rapa, B. oleracea and $N$. tabacum which were not analysed further in the original studies since they lack key CNGC domains $[18,21,22]$.

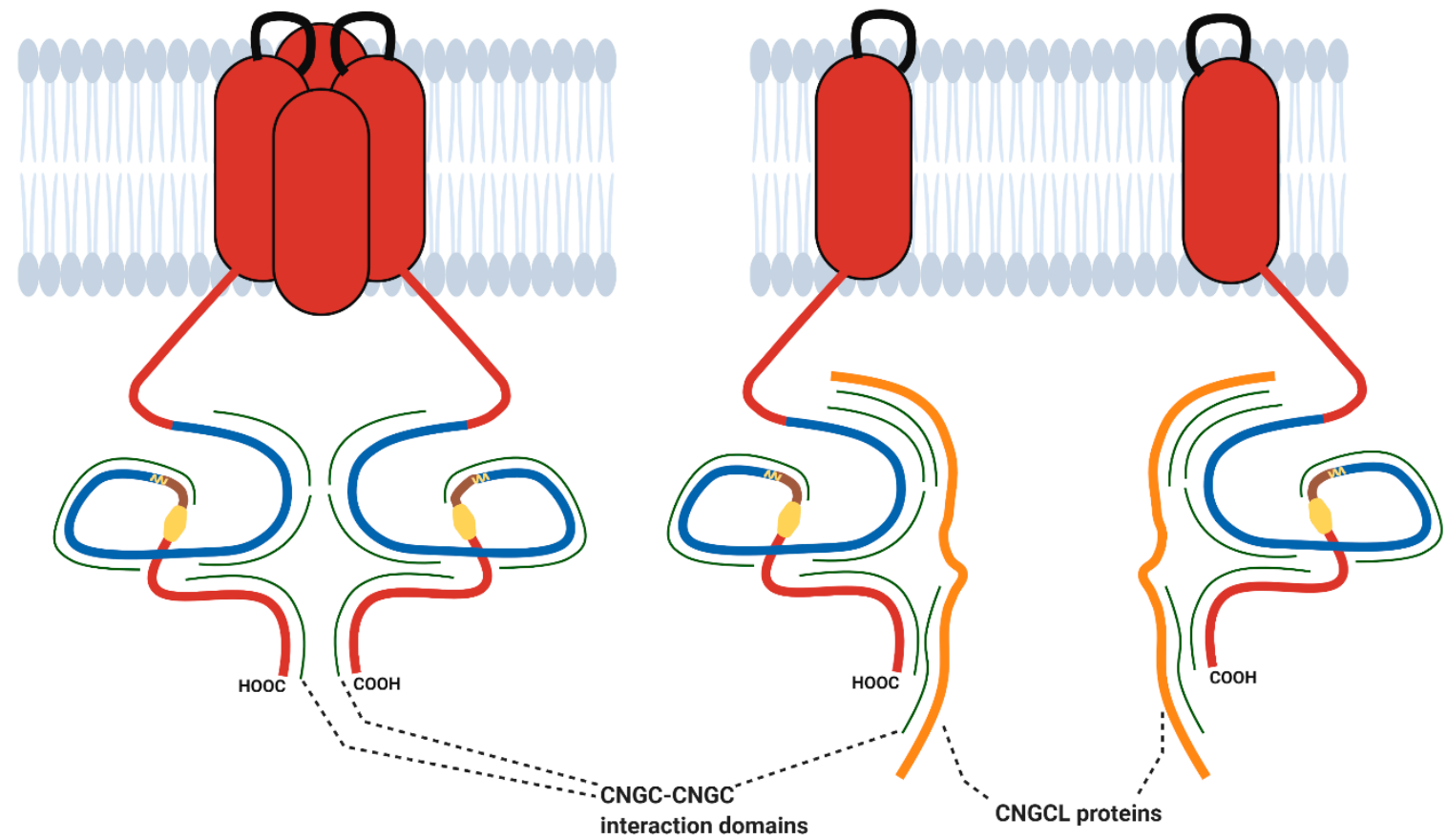

Figure 4. CNGCL proteins may disrupt the interactions between CNGC subunits and destabilise CNGC tetramers. For simplicity, pore loops and CT are shown in only two subunits. Figure created with BioRender.com.

We examined some of these CNGCL sequences in silico to determine whether they may have the potential to disrupt CNGC-CNGC interactions. Six CNGCL genes (Bra024083, Bo3g005110, Bo8g027170, Bo5g104990, Bo3g052670 and Bo6g074480) were identified from genome-wide analyses of B. rapa and B. oleracea and located in the EnsemblPlants database (https:/ / plants.ensembl.org/index.html) [22,23]. For the five B. oleracea genes, the protein sequences were extracted from their UniProtKB identifiers. For Bra024083, the annotation in EnsemblPlants predicts a 78 amino acid sequence, whereas the NCBI reference sequence for Bra024083, XP_009137913.1, predicts a 100 amino acid protein. Both sequences were used in the subsequent analysis. The seven protein sequences were used as queries to search for homologous sequences in the genomes of $B$. rapa and B. oleracea using the NCBI BLAST protein program with default parameters (https:/ /blast.ncbi.nlm.nih.gov/Blast. 
cgi?PAGE=Proteins). Following this, protein sequences of CNGCLs and the most similar CNGC identified in the BLAST search were submitted to pairwise local sequence using the EMBOSS Water program with default parameters (https:/ /www.ebi.ac.uk/Tools/psa/ emboss_water/). These alignments are presented in Figure A1 in Appendix A.

Bra024083 (NCBI reference sequence XP_009137913.1) was initially identified for its homology with AtCNGC17 [23]. In our analysis, the 78 amino acid prediction aligns to a region in the CT of BraCNGC17 (NCBI reference sequence XP_009127879.2, positions 640-728) with $76.4 \%$ identity. The 100 amino acid prediction aligns to an overlapping region of BraCNGC17 (positions 629-728) with 75\% identity. In B. oleracea, Bo3g005110 (UniProtKB_A0A0D3DKM8) and Bo8g027170 (UniProtKB_A0A0D3DKM8) are predicted to encode identical 99 amino acid peptides which align to a 70 amino acid stretch in the CT of BoCNGC7 (NCBI reference sequence XP_013585954.1, positions 579-648) with 80.0\% identity. Similarly, Bo5g104990 (UniProtKB_A0A0D3CHU3) is predicted to encode a 113 amino acid peptide which aligns to a 76 amino acid stretch in the CT of BoCNGC7 (positions 572-648) with 83.1\% identity. Bo3g052670 (UniProtKB_A0A0D3B8V0) is predicted to encode a 412 amino acid peptide which aligns to the NT sequence of BoCNGC12 (NCBI reference sequence XP_013631017.1, positions 1-425) with $68.2 \%$ identity and Bo6g074480 is predicted to encode a 480 amino acid peptide which aligns with BoCNGC12 (positions 103-648) with $60.9 \%$ identity. In silico observations may be misleading but these putative CNGCL proteins may warrant further attention to determine whether they are functional in planta and interact with CNGCs.

\section{Conclusions}

Research into plant CNGCs has historically focused on the role of individual CNGC genes. There is increasing evidence, however, to suggest that plant CNGCs function as heterotetrameric complexes.

To understand the role of CNGCs (whether in $\mathrm{Ca}^{2+}$ signalling or nutrition), it is necessary to determine which subunits interact and determine whether they form heterotetrameric complexes. If CNGC complexes were common, it will be important to determine which CNGC interactions occur preferentially and to study CNGC expression patterns to help predict the composition of CNGC complexes in different cell types. Consequently, a systematic study of CNGC interactions through BiFC and single-molecule fluorescence is needed to understand which complexes may be present in planta. In common with animal studies, cryo-electron microscopy should be able to resolve tetrameric structures. The advent of fluorophore-labelled cyclic nucleotides is now enabling the effect of cNMP binding on channel kinetics to be elucidated for animal homomeric and heterotetrameric CNGs [138] and could be applied to heterologously expressed plant CNGCs to further understand differences between complexes. It will subsequently be important to test the activities of these CNGCs in physiologically relevant conditions and determine how their behaviour is different from homotetrameric channels.

Recent studies, therefore, have significant implications for the future of CNGC research and may herald a major shift in our understanding of CNGC function. The role of CNGCs in plants may, truly, be complex.

Funding: This research was funded by the University of Cambridge Brookes and Isaac Newton Trusts and the University of Cambridge Commonwealth, European and International Trusts.

Institutional Review Board Statement: Not applicable.

Informed Consent Statement: Not applicable.

Data Availability Statement: Data can be obtained by contacting the corresponding author.

Conflicts of Interest: The authors declare no conflict of interest. 


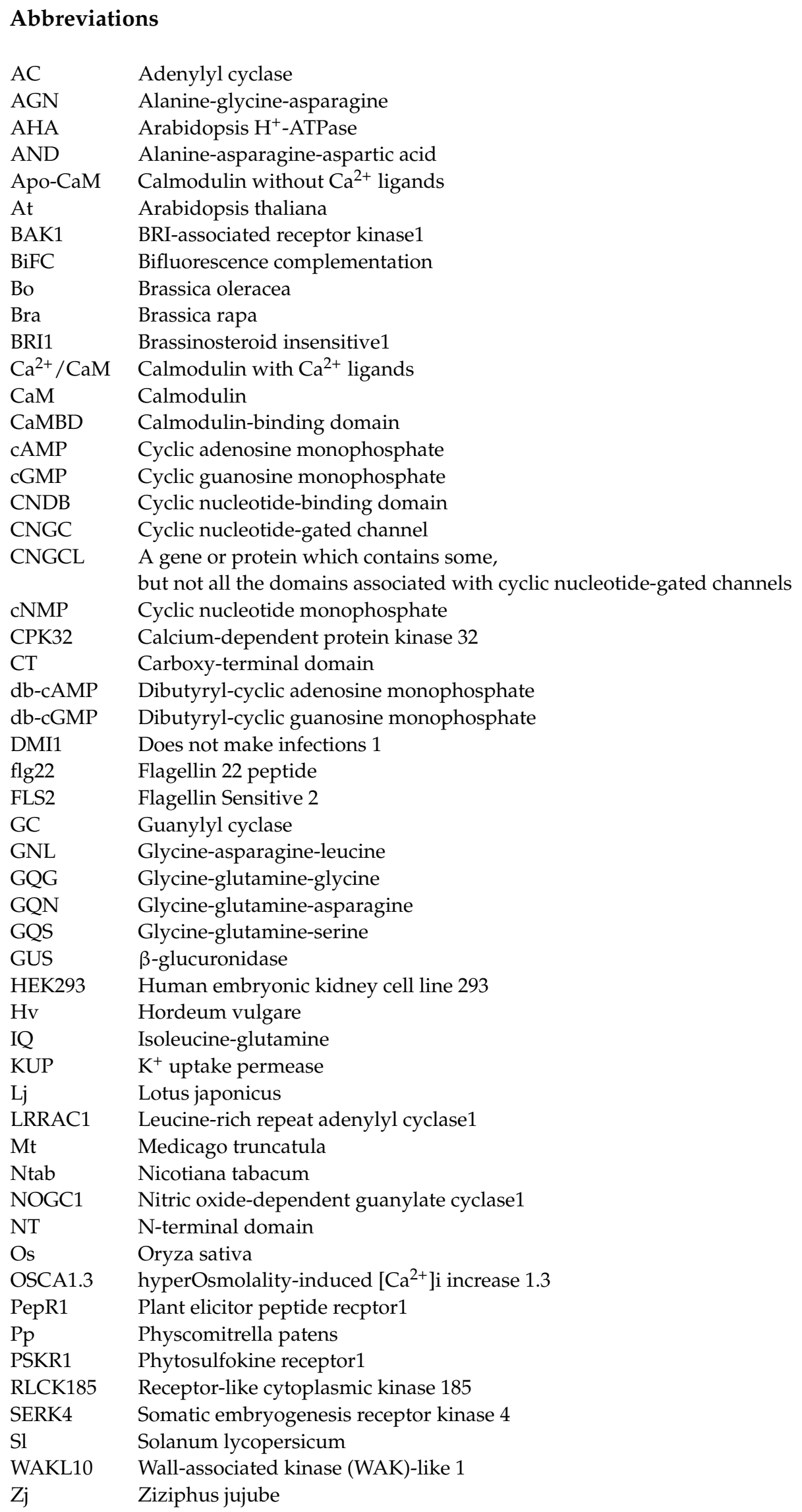




\section{Appendix A}

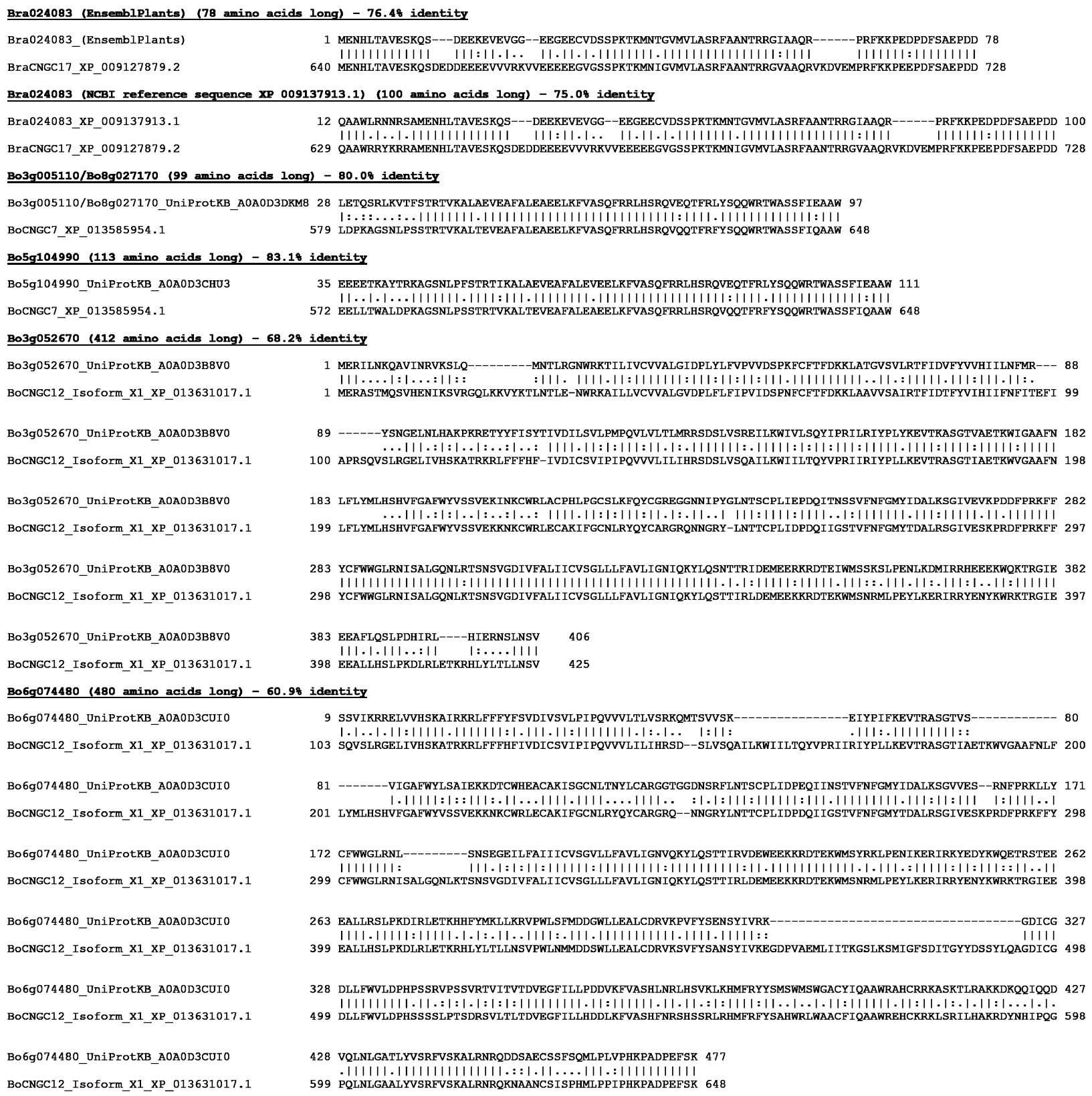

Figure A1. Local sequence alignments of CNGCL protein sequences against CNGC protein sequences. In each case the CNGCL sequence is displayed above the CNGC sequence. In each subheading the full length of the CNGCL sequence is given as well as the percentage identity with the CNGC sequence shown.

\section{References}

1. Leng, Q.; Mercier, R.W.; Yao, W.; Berkowitz, G.A. Cloning and first functional characterization of a plant cyclic nucleotide-gated cation channel. Plant Physiol. 1999, 121, 753-761. [CrossRef] [PubMed]

2. Leng, Q.; Mercier, R.W.; Hua, B.-G.; Fromm, H.; Berkowitz, G.A. Electrophysiological analysis of cloned cyclic nucleotide-gated ion channels. Plant Physiol. 2002, 128, 400-410. [CrossRef] [PubMed]

3. Qi, Z.; Verma, R.; Gehring, C.; Yamaguchi, Y.; Zhao, Y.; Ryan, C.A.; Berkowitz, G.A. Ca ${ }^{2+}$ signaling by plant Arabidopsis thaliana Pep peptides depends on AtPepR1, a receptor with guanylyl cyclase activity, and cGMP-activated Ca ${ }^{2+}$ channels. Proc. Natl. Acad. Sci. USA 2010, 107, 21193-21198. [CrossRef] [PubMed]

4. Wang, Y.-F.; Munemasa, S.; Nishimura, N.; Ren, H.-M.; Robert, N.; Han, M.; Puzõrjova, I.; Kollist, H.; Lee, S.; Mori, I.; et al. Identification of cyclic GMP-activated nonselective $\mathrm{Ca}^{2+}$-permeable cation channels and associated CNGC5 and CNGC6 genes in Arabidopsis guard cells. Plant Physiol. 2013, 163, 578-590. [CrossRef] 
5. Gao, Q.-F.; Fei, C.-F.; Dong, J.-Y.; Gu, L.-L.; Wang, Y.-F. Arabidopsis CNGC18 is a $\mathrm{Ca}^{2+}$-permeable channel. Mol. Plant 2014, 7, 739-743. [CrossRef] [PubMed]

6. Gao, Q.-F.; Gu, L.-L.; Wang, H.-Q.; Fei, C.-F.; Fang, X.; Hussain, J.; Sun, S.-J.; Dong, J.-Y.; Liu, H.; Wang, Y.-F. Cyclic nucleotidegated channel 18 is an essential $\mathrm{Ca}^{2+}$ channel in pollen tube tips for pollen tube guidance to ovules in Arabidopsis. Proc. Natl. Acad. Sci. USA 2016, 113, 3096-3101. [CrossRef]

7. Mori, I.C.; Nobukiyo, Y.; Nakahara, Y.; Shibasaka, M.; Furuichi, T.; Katsuhara, M. A cyclic nucleotide-gated channel, HvCNGC2-3, is activated by the co-presence of $\mathrm{Na}^{+}$and $\mathrm{K}^{+}$and permeable to $\mathrm{Na}^{+}$and $\mathrm{K}^{+}$non-selectively. Plants 2018, 7. [CrossRef]

8. Zhang, Z.; Hou, C.; Tian, W.; Li, L.; Zhu, H. Electrophysiological studies revealed CaM1-mediated regulation of the Arabidopsis calcium channel CNGC12. Front. Plant Sci. 2019, 10. [CrossRef]

9. Tian, W.; Wang, C.; Gao, Q.; Li, L.; Luan, S. Calcium spikes, waves and oscillations in plant development and biotic interactions. Nat. Plants 2020, 6, 750-759. [CrossRef]

10. Dietrich, P.; Moeder, W.; Yoshioka, K. Plant cyclic nucleotide-gated channels: New insights on their functions and regulation. Plant Physiol. 2020, 184, 27-38. [CrossRef]

11. Blanco, E.; Fortunato, S.; Viggiano, L.; de Pinto, M.C. Cyclic AMP: A polyhedral signalling molecule in plants. Int. J. Mol. Sci. 2020, 21. [CrossRef] [PubMed]

12. Chin, K.; Moeder, W.; Yoshioka, K. Biological roles of cyclic-nucleotide-gated ion channels in plants: What we know and don't know about this 20 member ion channel family. Botany 2009, 87, 668-677. [CrossRef]

13. Duszyn, M.; Świeżawska, B.; Szmidt-Jaworska, A.; Jaworski, K. Cyclic nucleotide gated channels (CNGCs) in plant signallingCurrent knowledge and perspectives. Plant Physiol. 2019, 241. [CrossRef] [PubMed]

14. Jha, S.K.; Sharma, M.; Pandey, G.K. Role of cyclic nucleotide gated channels in stress management in plants. Curr. Genom. 2016, 17, 315-329. [CrossRef] [PubMed]

15. Moeder, W.; Urquhart, W.; Ung, H.; Yoshioka, K. The role of cyclic nucleotide-gated ion channels in plant immunity. Mol. Plant 2011, 4, 442-452. [CrossRef] [PubMed]

16. Mäser, P.; Thomine, S.; Schroeder, J.I.; Ward, J.M.; Hirschi, K.; Sze, H.; Talke, I.N.; Amtmann, A.; Maathuis, F.J.; Sanders, D.; et al. Phylogenetic relationships within cation transporter families of Arabidopsis. Plant Physiol. 2001, 126, 1646-1667. [CrossRef]

17. Zeng, H.; Zhao, B.; Wu, H.; Zhu, Y.; Chen, H. Comprehensive in silico characterization and expression profiling of nine gene families associated with calcium transport in soybean. Agronomy 2020, 10. [CrossRef]

18. Nawaz, Z.; Kakar, K.U.; Ullah, R.; Yu, S.; Zhang, J.; Shu, Q.-Y.; Ren, X. Genome-wide identification, evolution and expression analysis of cyclic nucleotide-gated channels in tobacco (Nicotiana tabacum L.). Genomics 2019, 111, 142-158. [CrossRef]

19. Nawaz, Z.; Kakar, K.U.; Saand, M.A.; Shu, Q.-Y. Cyclic nucleotide-gated ion channel gene family in rice, identification, characterization and experimental analysis of expression response to plant hormones, biotic and abiotic stresses. BMC Genom. 2014, 15, 1-18. [CrossRef]

20. Guo, J.; Islam, M.A.; Lin, H.; Ji, C.; Duan, Y.; Liu, P.; Zeng, Q.; Day, B.; Kang, Z.; Guo, J. Genome-wide identification of cyclic nucleotide-gated ion channel gene family in wheat and functional analyses of TaCNGC14 and TaCNGC16. Front. Plant Sci. 2018, 9, 18. [CrossRef]

21. Hao, L.; Qiao, X. Genome-wide identification and analysis of the CNGC gene family in maize. PeerJ 2018, 6, e5816. [CrossRef] [PubMed]

22. Kakar, K.U.; Nawaz, Z.; Kakar, K.; Ali, E.; Almoneafy, A.A.; Ullah, R.; Ren, X.; Shu, Q.-Y. Comprehensive genomic analysis of the CNGC gene family in Brassica oleracea: Novel insights into synteny, structures, and transcript profiles. BMC Genom. 2017, 18, 1-18. [CrossRef] [PubMed]

23. Li, Q.; Yang, S.; Ren, J.; Ye, X.; jiang, X.; Liu, Z. Genome-wide identification and functional analysis of the cyclic nucleotide-gated channel gene family in Chinese cabbage. 3 Biotech 2019, 9, 1-14. [CrossRef] [PubMed]

24. Ma, W.; Ali, R.; Berkowitz, G.A. Characterization of plant phenotypes associated with loss-of-function of AtCNGC1, a plant cyclic nucleotide gated cation channel. Plant Physiol. Biochem. 2006, 44, 494-505. [CrossRef] [PubMed]

25. Moon, J.Y.; Belloeil, C.; Ianna, M.L.; Shin, R. Arabidopsis CNGC family members contribute to heavy metal ion uptake in plants. Int. J. Mol. Sci. 2019, 20, 413. [CrossRef] [PubMed]

26. Sunkar, R.; Kaplan, B.; Bouché, N.; Arazi, T.; Dolev, D.; Talke, I.N.; Maathuis, F.J.M.; Sanders, D.; Bouchez, D.; Fromm, H. Expression of a truncated tobacco NtCBP4 channel in transgenic plants and disruption of the homologous Arabidopsis CNGC1 gene confer $\mathrm{Pb}^{2+}$ tolerance. Plant J. 2000, 24, 533-542. [CrossRef] [PubMed]

27. Chin, K.; DeFalco, T.A.; Moeder, W.; Yoshioka, K. The Arabidopsis cyclic nucleotide-gated ion channels AtCNGC2 and AtCNGC4 work in the same signaling pathway to regulate pathogen defense and floral transition. Plant Physiol. 2013, 163, 611-624. [CrossRef]

28. Finka, A.; Cuendet, A.F.H.; Maathuis, F.J.M.; Saidi, Y.; Goloubinoff, P. Plasma membrane cyclic nucleotide gated calcium channels control land plant thermal sensing and acquired thermotolerance. Plant Cell 2012, 24, 3333-3348. [CrossRef]

29. Tian, W.; Hou, C.; Ren, Z.; Wang, C.; Zhao, F.; Dahlbeck, D.; Hu, S.; Zhang, L.; Niu, Q.; Li, L.; et al. A calmodulin-gated calcium channel links pathogen patterns to plant immunity. Nature 2019, 572, 131-135. [CrossRef]

30. Ali, R.; Ma, W.; Lemtiri-Chlieh, F.; Tsaltas, D.; Leng, Q.; von Bodman, S.; Berkowitz, G.A. Death don't have no mercy and neither does calcium: Arabidopsis CYCLIC NUCLEOTIDE GATED CHANNEL2 and innate immunity. Plant Cell 2007, 19, 1081-1095. [CrossRef] 
31. Clough, S.J.; Fengler, K.A.; Yu, I.; Lippok, B.; Smith, R.K.; Bent, A.F. The Arabidopsis dnd1 "defense, no death" gene encodes a mutated cyclic nucleotide-gated ion channel. Proc. Natl. Acad. Sci. USA 2000, 97, 9323-9328. [CrossRef] [PubMed]

32. Cui, Y.; Lu, S.; Li, Z.; Cheng, J.; Hu, P.; Zhu, T.; Wang, X.; Jin, M.; Wang, X.; Li, L.; et al. CYCLIC NUCLEOTIDE-GATED ION CHANNELs 14 and 16 promote tolerance to heat and chilling in rice. Plant Physiol. 2020, 183, 1794-1808. [CrossRef] [PubMed]

33. Fischer, C.; DeFalco, T.A.; Karia, P.; Snedden, W.A.; Moeder, W.; Yoshioka, K.; Dietrich, P. Calmodulin as a Ca ${ }^{2+}$-sensing subunit of Arabidopsis cyclic nucleotide-gated channel complexes. Plant Cell Physiol. 2017, 58, 1208-1221. [CrossRef] [PubMed]

34. Genger, R.K.; Jurkowski, G.I.; McDowell, J.M.; Lu, H.; Jung, H.W.; Greenberg, J.T.; Bent, A.F. Signaling pathways that regulate the enhanced disease resistance of Arabidopsis "Defense, No Death" mutants. MPMI 2008, 21, 1285-1296. [CrossRef]

35. Lu, M.; Zhang, Y.; Tang, S.; Pan, J.; Yu, Y.; Han, J.; Li, Y.; Du, X.; Nan, Z.; Sun, Q. AtCNGC2 is involved in jasmonic acid-induced calcium mobilization. J. Exp. Bot. 2016, 67, 809-819. [CrossRef]

36. Ma, W.; Qi, Z.; Smigel, A.; Walker, R.K.; Verma, R.; Berkowitz, G.A. Ca ${ }^{2+}$, cAMP, and transduction of non-self perception during plant immune responses. Proc. Natl. Acad. Sci. USA 2009, 106, 20995-21000. [CrossRef]

37. Ma, W.; Smigel, A.; Walker, R.K.; Moeder, W.; Yoshioka, K.; Berkowitz, G.A. Leaf senescence signaling: The Ca ${ }^{2+}$-conducting Arabidopsis cyclic nucleotide gated channel2 acts through nitric oxide to repress senescence programming. Plant Physiol. 2010, 154, 733-743. [CrossRef]

38. Wang, Y.; Kang, Y.; Ma, C.; Miao, R.; Wu, C.; Long, Y.; Ge, T.; Wu, Z.; Hou, X.; Zhang, J.; et al. CNGC2 is a Ca ${ }^{2+}$ influx channel that prevents accumulation of apoplastic $\mathrm{Ca}^{2+}$ in the leaf. Plant Physiol. 2017, 173, 1342-1354. [CrossRef]

39. Yu, I.; Parker, J.; Bent, A.F. Gene-for-gene disease resistance without the hypersensitive response in Arabidopsis dnd1 mutant. Proc. Natl. Acad. Sci. USA 1998, 95, 7819-7824. [CrossRef]

40. Gobert, A.; Park, G.; Amtmann, A.; Sanders, D.; Maathuis, F.J.M. Arabidopsis thaliana Cyclic Nucleotide Gated Channel 3 forms a non-selective ion transporter involved in germination and cation transport. J. Exp. Bot. 2006, 57, 791-800. [CrossRef]

41. Balagué, C.; Lin, B.; Alcon, C.; Flottes, G.; Malmström, S.; Köhler, C.; Neuhaus, G.; Pelletier, G.; Gaymard, F.; Roby, D. HLM1, an essential signaling component in the hypersensitive response, is a member of the cyclic nucleotide-gated channel ion channel family. Plant Cell 2003, 15, 365-379. [CrossRef] [PubMed]

42. Massange-Sánchez, J.A.; Palmeros-Suárez, P.A.; Espitia-Rangel, E.; Rodríguez-Arévalo, I.; Sánchez-Segura, L.; Martínez-Gallardo, N.A.; Alatorre-Cobos, F.; Tiessen, A.; Délano-Frier, J.P. Overexpression of Grain Amaranth (Amaranthus hypochondriacus) AhERF or AhDOF transcription factors in Arabidopsis thaliana increases water deficit- and salt-stress tolerance, respectively, via contrasting stress-amelioration mechanisms. PLoS ONE 2016, 11. [CrossRef] [PubMed]

43. Tan, Y.-Q.; Yang, Y.; Zhang, A.; Fei, C.-F.; Gu, L.-L.; Sun, S.-J.; Xu, W.; Wang, L.; Liu, H.; Wang, Y.-F. Three CNGC family members, CNGC5, CNGC6, and CNGC9, are required for constitutive growth of Arabidopsis root hairs as $\mathrm{Ca}^{2+}$-permeable channels. Plant Commun. 2020, 1, 100001. [CrossRef] [PubMed]

44. Brost, C.; Studtrucker, T.; Reimann, R.; Denninger, P.; Czekalla, J.; Krebs, M.; Fabry, B.; Schumacher, K.; Grossmann, G.; Dietrich, P. Multiple cyclic nucleotide-gated channels coordinate calcium oscillations and polar growth of root hairs. Plant J. 2019, 99, 910-923. [CrossRef] [PubMed]

45. Gao, F.; Han, X.; Wu, J.; Zheng, S.; Shang, Z.; Sun, D.; Zhou, R.; Li, B. A heat-activated calcium-permeable channel-Arabidopsis cyclic nucleotide-gated ion channel 6-Is involved in heat shock responses. Plant J. 2012, 70, 1056-1069. [CrossRef] [PubMed]

46. Pan, Y.; Chai, X.; Gao, Q.; Zhou, L.; Zhang, S.; Li, L.; Luan, S. Dynamic interactions of plant CNGC subunits and calmodulins drive oscillatory $\mathrm{Ca}^{2+}$ channel activities. Dev. Cell 2019, 48, 710-725. [CrossRef]

47. Tunc-Ozdemir, M.; Rato, C.; Brown, E.; Rogers, S.; Mooneyham, A.; Frietsch, S.; Myers, C.T.; Poulsen, L.R.; Malhó, R.; Harper, J.F. Cyclic nucleotide gated channels 7 and 8 are essential for male reproductive fertility. PLoS ONE 2013, 8, e55277. [CrossRef]

48. Borsics, T.; Webb, D.; Andeme-Ondzighi, C.; Staehelin, L.A.; Christopher, D.A. The cyclic nucleotide-gated calmodulin-binding channel AtCNGC10 localizes to the plasma membrane and influences numerous growth responses and starch accumulation in Arabidopsis thaliana. Planta 2007, 225, 563-573. [CrossRef]

49. Jin, Y.; Jing, W.; Zhang, Q.; Zhang, W. Cyclic nucleotide gated channel 10 negatively regulates salt tolerance by mediating Na ${ }^{+}$ transport in Arabidopsis. J. Plant Res. 2015, 128, 211-220. [CrossRef]

50. DeFalco, T.A.; Marshall, C.B.; Munro, K.; Kang, H.-G.; Moeder, W.; Ikura, M.; Snedden, W.A.; Yoshioka, K. Multiple calmodulinbinding sites positively and negatively regulate Arabidopsis CYCLIC NUCLEOTIDE-GATED CHANNEL12. Plant Cell 2016, 28, 1738-1751. [CrossRef]

51. Urquhart, W.; Gunawardena, A.H.L.A.N.; Moeder, W.; Ali, R.; Berkowitz, G.A.; Yoshioka, K. The chimeric cyclic nucleotide-gated ion channel ATCNGC11/12 constitutively induces programmed cell death in a $\mathrm{Ca}^{2+}$ dependent manner. Plant Mol. Biol. 2007, 65, 747-761. [CrossRef] [PubMed]

52. Yoshioka, K.; Kachroo, P.; Tsui, F.; Sharma, S.B.; Shah, J.; Klessig, D.F. Environmentally sensitive, SA-dependent defense responses in the cpr22 mutant of Arabidopsis. Plant J. 2001, 26, 447-459. [CrossRef] [PubMed]

53. Yoshioka, K.; Moeder, W.; Kang, H.-G.; Kachroo, P.; Masmoudi, K.; Berkowitz, G.; Klessig, D.F. The chimeric Arabidopsis CYCLIC NUCLEOTIDE-GATED ION CHANNEL11/12 activates multiple pathogen resistance responses. Plant Cell 2006, 18, 747-763. [CrossRef] [PubMed]

54. Dindas, J.; Scherzer, S.; Roelfsema, M.R.G.; von Meyer, K.; Müller, H.M.; Al-Rasheid, K.A.S.; Palme, K.; Dietrich, P.; Becker, D.;

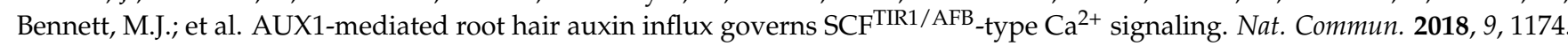
[CrossRef] 
55. Shih, H.-W.; DePew, C.L.; Miller, N.D.; Monshausen, G.B. The cyclic nucleotide-gated channel CNGC14 regulates root gravitropism in Arabidopsis thaliana. Curr. Biol. 2015, 25, 3119-3125. [CrossRef] [PubMed]

56. Zeb, Q.; Wang, X.; Hou, C.; Zhang, X.; Dong, M.; Zhang, S.; Zhang, Q.; Ren, Z.; Tian, W.; Zhu, H.; et al. The interaction of CaM7 and CNGC14 regulates root hair growth in Arabidopsis. J. Integr. Plant Biol. 2020, 62, 887-896. [CrossRef]

57. Zhang, S.; Pan, Y.; Tian, W.; Dong, M.; Zhu, H.; Luan, S.; Li, L. Arabidopsis CNGC14 mediates calcium influx required for tip growth in root hairs. Mol. Plant 2017, 10, 1004-1006. [CrossRef]

58. Leitão, N.; Dangeville, P.; Carter, R.; Charpentier, M. Nuclear calcium signatures are associated with root development. Nat. Commun. 2019, 10, 1-9. [CrossRef]

59. Tunc-Ozdemir, M.; Tang, C.; Ishka, M.R.; Brown, E.; Groves, N.R.; Myers, C.T.; Rato, C.; Poulsen, L.R.; McDowell, S.; Miller, G.; et al. A cyclic nucleotide-gated channel (CNGC16) in pollen is critical for stress tolerance in pollen reproductive development. Plant Physiol. 2013, 161, 1010-1020. [CrossRef] [PubMed]

60. Ladwig, F.; Dahlke, R.I.; Stührwohldt, N.; Hartmann, J.; Harter, K.; Sauter, M. Phytosulfokine regulates growth in Arabidopsis through a response module at the plasma membrane that includes CYCLIC NUCLEOTIDE-GATED CHANNEL17, $\mathrm{H}^{+}$-ATPase, and BAK1. Plant Cell 2015, 27, 1718-1729. [CrossRef]

61. Chang, F.; Yan, A.; Zhao, L.-N.; Wu, W.-H.; Yang, Z. A putative calcium-permeable cyclic nucleotide-gated channel, CNGC18, regulates polarized pollen tube growth. J. Integr. Plant Biol. 2007, 49, 1261-1270. [CrossRef]

62. Frietsch, S.; Wang, Y.-F.; Sladek, C.; Poulsen, L.R.; Romanowsky, S.M.; Schroeder, J.I.; Harper, J.F. A cyclic nucleotide-gated channel is essential for polarized tip growth of pollen. Proc. Natl. Acad. Sci. USA 2007, 104, 14531-14536. [CrossRef] [PubMed]

63. Zhou, L.; Lan, W.; Jiang, Y.; Fang, W.; Luan, S. A calcium-dependent protein kinase interacts with and activates a calcium channel to regulate pollen tube growth. Mol. Plant 2014, 7, 369-376. [CrossRef] [PubMed]

64. Jogawat, A.; Meena, M.K.; Kundu, A.; Varma, M.; Vadassery, J. Calcium channel CNGC19 mediates basal defense signaling to regulate colonization by Piriformospora indica in Arabidopsis roots. J. Exp. Bot. 2020, 71, 2752-2768. [CrossRef] [PubMed]

65. Kugler, A.; Köhler, B.; Palme, K.; Wolff, P.; Dietrich, P. Salt-dependent regulation of a CNG channel subfamily in Arabidopsis. BMC Plant Biol. 2009, 9, 140. [CrossRef] [PubMed]

66. Meena, M.K.; Prajapati, R.; Krishna, D.; Divakaran, K.; Pandey, Y.; Reichelt, M.; Mathew, M.K.; Boland, W.; Mithöfer, A.; Vadassery, J. The $\mathrm{Ca}^{2+}$ channel CNGC19 regulates Arabidopsis defense against spodoptera herbivory. Plant Cell 2019, 31, 1539-1562. [CrossRef] [PubMed]

67. Yu, X.; Xu, G.; Li, B.; de Souza Vespoli, L.; Liu, H.; Moeder, W.; Chen, S.; de Oliveira, M.V.V.; Ariádina de Souza, S.; Shao, W.; et al. The receptor kinases BAK1/SERK4 regulate $\mathrm{Ca}^{2+}$ channel-mediated cellular homeostasis for cell death containment. Curr. Biol. 2019, 29, 3778-3790. [CrossRef]

68. Kaupp, U.B.; Seifert, R. Cyclic nucleotide-gated ion channels. Physiol. Rev. 2002, 82, 769-824. [CrossRef]

69. Liu, D.T.; Tibbs, G.R.; Siegelbaum, S.A. Subunit stoichiometry of cyclic nucleotide-gated channels and effects of subunit order on channel function. Neuron 1996, 16, 983-990. [CrossRef]

70. Michalakis, S.; Becirovic, E.; Biel, M. Retinal cyclic nucleotide-gated channels: From pathophysiology to therapy. Int. J. Mol. Sci. 2018, 19, 749. [CrossRef]

71. Shuart, N.G.; Haitin, Y.; Camp, S.S.; Black, K.D.; Zagotta, W.N. Molecular mechanism for 3:1 subunit stoichiometry of rod cyclic nucleotide-gated ion channels. Nat. Commun. 2011, 2, 457. [CrossRef]

72. Dreyer, I.; Porée, F.; Schneider, A.; Mittelstädt, J.; Bertl, A.; Sentenac, H.; Thibaud, J.-B.; Mueller-Roeber, B. Assembly of plant Shaker-like $\mathrm{K}_{\text {out }}$ channels requires two distinct sites of the channel $\alpha$-subunit. Biophys. J. 2004, 87, 858-872. [CrossRef] [PubMed]

73. Lebaudy, A.; Pascaud, F.; Véry, A.-A.; Alcon, C.; Dreyer, I.; Thibaud, J.-B.; Lacombe, B. Preferential KAT1-KAT2 heteromerization determines inward $\mathrm{K}^{+}$current properties in Arabidopsis guard cells. J. Biol. Chem. 2010, 285, 6265-6274. [CrossRef] [PubMed]

74. Naso, A.; Dreyer, I.; Pedemonte, L.; Testa, I.; Gomez-Porras, J.L.; Usai, C.; Mueller-Rueber, B.; Diaspro, A.; Gambale, F.; Picco, C. The role of the C-terminus for functional heteromerization of the plant channel KDC1. Biophys. J. 2009, 96, 4063-4074. [CrossRef] [PubMed]

75. Nieves-Cordones, M.; Chavanieu, A.; Jeanguenin, L.; Alcon, C.; Szponarski, W.; Estaran, S.; Chérel, I.; Zimmermann, S.; Sentenac, H.; Gaillard, I. Distinct amino acids in the C-linker domain of the Arabidopsis $\mathrm{K}^{+}$channel KAT2 determine its subcellular localization and activity at the plasma membrane. Plant Physiol. 2014, 164, 1415-1429. [CrossRef] [PubMed]

76. Wang, X.-P.; Chen, L.-M.; Liu, W.-X.; Shen, L.-K.; Wang, F.-L.; Zhou, Y.; Zhang, Z.; Wu, W.-H.; Wang, Y. AtKC1 and CIPK23 synergistically modulate AKT1-mediated low-potassium stress responses in Arabidopsis. Plant Physiol. 2016, 170, $2264-2277$. [CrossRef]

77. Charpentier, M.; Sun, J.; Martins, T.V.; Radhakrishnan, G.V.; Findlay, K.; Soumpourou, E.; Thouin, J.; Véry, A.-A.; Sanders, D.; Morris, R.J.; et al. Nuclear-localized cyclic nucleotide-gated channels mediate symbiotic calcium oscillations. Science 2016, 352, 1102-1105. [CrossRef] [PubMed]

78. Zelman, A.K.; Dawe, A.; Gehring, C.; Berkowitz, G.A. Evolutionary and structural perspectives of plant cyclic nucleotide-gated cation channels. Front. Plant Sci. 2012, 3, 95. [CrossRef]

79. Wang, L.; Li, M.; Liu, Z.; Dai, L.; Zhang, M.; Wang, L.; Zhao, J.; Liu, M. Genome-wide identification of CNGC genes in Chinese jujube (Ziziphus jujuba Mill.) and ZjCNGC2 mediated signalling cascades in response to cold stress. BMC Genomics 2020, 21, 191-216. [CrossRef] 
80. Christopher, D.A.; Borsics, T.; Yuen, C.Y.; Ullmer, W.; Andème-Ondzighi, C.; Andres, M.A.; Kang, B.-H.; Staehelin, L.A. The cyclic nucleotide gated cation channel AtCNGC10 traffics from the ER via Golgi vesicles to the plasma membrane of Arabidopsis root and leaf cells. BMC Plant Biol. 2007, 7, 48. [CrossRef]

81. Baxter, J.; Moeder, W.; Urquhart, W.; Shahinas, D.; Chin, K.; Christendat, D.; Kang, H.-G.; Angelova, M.; Kato, N.; Yoshioka, $\mathrm{K}$. Identification of a functionally essential amino acid for Arabidopsis cyclic nucleotide gated ion channels using the chimeric AtCNGC11/12 gene. Plant J. 2008, 56, 457-469. [CrossRef] [PubMed]

82. Fischer, C.; Kugler, A.; Hoth, S.; Dietrich, P. An IQ domain mediates the interaction with calmodulin in a plant cyclic nucleotidegated channel. Plant Cell Physiol. 2013, 54, 573-584. [CrossRef] [PubMed]

83. Yuen, C.C.Y.; Christopher, D.A. The group IV-A cyclic nucleotide-gated channels, CNGC19 and CNGC20, localize to the vacuole membrane in Arabidopsis thaliana. AoB PLANTS 2013, 5. [CrossRef]

84. Lemtiri-Chlieh, F.; Berkowitz, G.A. Cyclic adenosine monophosphate regulates calcium channels in the plasma membrane of Arabidopsis leaf guard and mesophyll cells. J. Biol. Chem. 2004, 279, 35306-35312. [CrossRef]

85. Hua, B.-G.; Mercier, R.W.; Leng, Q.; Berkowitz, G.A. Plants do it differently. A new basis for potassium/sodium selectivity in the pore of an ion channel. Plant Physiol. 2003, 132, 1353-1361. [CrossRef]

86. Mercier, R.W.; Rabinowitz, N.M.; Ali, R.; Gaxiola, R.A.; Berkowitz, G.A. Yeast hygromycin sensitivity as a functional assay of cyclic nucleotide gated cation channels. Plant Physiol. Biochem. 2004, 42, 529-536. [CrossRef]

87. Ali, R.; Zielinski, R.E.; Berkowitz, G.A. Expression of plant cyclic nucleotide-gated cation channels in yeast. J. Exp. Bot. 2006, 57, 125-138. [CrossRef]

88. Li, X.; Borsics, T.; Harrington, H.M.; Christopher, D.A. Arabidopsis AtCNGC10 rescues potassium channel mutants of E. coli, yeast and Arabidopsis and is regulated by calcium/calmodulin and cyclic GMP in E. coli. Funct. Plant Biol. 2005, 32, 643-653. [CrossRef]

89. Wang, J.; Liu, X.; Zhang, A.; Ren, Y.; Wu, F.; Wang, G.; Xu, Y.; Lei, C.; Zhu, S.; Pan, T.; et al. A cyclic nucleotide-gated channel mediates cytoplasmic calcium elevation and disease resistance in rice. Cell Res. 2019, 29, 820-831. [CrossRef]

90. Xu, Y.; Yang, J.; Wang, Y.; Wang, J.; Yu, Y.; Long, Y.; Wang, Y.; Zhang, H.; Ren, Y.; Chen, J.; et al. OsCNGC13 promotes seed-setting rate by facilitating pollen tube growth in stylar tissues. PLoS Genet. 2017, 13, e1006906. [CrossRef]

91. Schuurink, R.C.; Shartzer, S.F.; Fath, A.; Jones, R.L. Characterization of a calmodulin-binding transporter from the plasma membrane of barley aleurone. Proc. Natl. Acad. Sci. USA 1998, 95, 1944-1949. [CrossRef] [PubMed]

92. Köhler, C.; Merkle, T.; Neuhaus, G. Characterisation of a novel gene family of putative cyclic nucleotide- and calmodulin-regulated ion channels in Arabidopsis thaliana. Plant J. 1999, 18, 97-104. [CrossRef] [PubMed]

93. Arazi, T.; Sunkar, R.; Kaplan, B.; Fromm, H. A tobacco plasma membrane calmodulin-binding transporter confers $\mathrm{Ni}^{2+}$ tolerance and $\mathrm{Pb}^{2+}$ hypersensitivity in transgenic plants. Plant J. 1999, 20, 171-182. [CrossRef] [PubMed]

94. Zheng, X.; Fu, Z.; Su, D.; Zhang, Y.; Li, M.; Pan, Y.; Li, H.; Li, S.; Grassucci, R.A.; Ren, Z.; et al. Mechanism of ligand activation of a eukaryotic cyclic nucleotide-gated channel. Nat. Struct. Biol. 2020, 27, 625-634. [CrossRef]

95. Jammes, F.; Hu, H.-C.; Villiers, F.; Bouten, R.; Kwak, J.M. Calcium-permeable channels in plant cells. FEBS J. 2011, $278,4262-4276$. [CrossRef]

96. Lemtiri-Chlieh, F.; Arold, S.T.; Gehring, C. $\mathrm{Mg}^{2+}$ is a missing link in plant cell $\mathrm{Ca}^{2+}$ signalling and homeostasis-a study on Vicia faba guard cells. Int. J. Mol. Sci. 2020, 21, 3771. [CrossRef]

97. Hua, B.-G.; Mercier, R.W.; Zielinski, R.E.; Berkowitz, G.A. Functional interaction of calmodulin with a plant cyclic nucleotide gated cation channel. Plant Physiol. Biochem. 2003, 41, 945-954. [CrossRef]

98. Zagotta, W.N.; Siegelbaum, S.A. Structure and function of cyclic nucleotide-gated channels. Annu. Rev. Neurosci. 1996, 19, 235-263. [CrossRef]

99. Demidchik, V.; Straltsova, D.; Medvedev, S.S.; Pozhvanov, G.A.; Sokolik, A.; Yurin, V. Stress-induced electrolyte leakage: The role of $\mathrm{K}^{+}$-permeable channels and involvement in programmed cell death and metabolic adjustment. J. Exp. Bot. 2014, 65, 1259-1270. [CrossRef]

100. Demidchik, V.; Shabala, S.N.; Coutts, K.B.; Tester, M.A.; Davies, J.M. Free oxygen radicals regulate plasma membrane Ca ${ }^{2+}$ - and $\mathrm{K}^{+}$-permeable channels in plant root cells. J. Cell Sci. 2003, 116, 81-88. [CrossRef]

101. Kaplan, B.; Sherman, T.; Fromm, H. Cyclic nucleotide-gated channels in plants. FEBS Lett. 2007, 581, 2237-2246. [CrossRef] [PubMed]

102. Isner, J.-C.; Maathuis, F.J.M. cGMP signalling in plants: From enigma to main stream. Funct. Plant Biol. 2018, 45, 93-101. [CrossRef] [PubMed]

103. Świeżawska, B.; Duszyn, M.; Jaworski, K.; Szmidt-Jaworska, A. Downstream targets of cyclic nucleotides in plants. Front. Plant Sci. 2018, 9, 1428. [CrossRef] [PubMed]

104. Gross, I.; Durner, J. In search of enzymes with a role in $3^{\prime}, 5^{\prime}$-cyclic guanosine monophosphate metabolism in plants. Front. Plant Sci. 2016, 7, 576. [CrossRef] [PubMed]

105. Al-Younis, I.; Wong, A.; Gehring, C. The Arabidopsis thaliana $\mathrm{K}^{+}$-uptake permease 7 (AtKUP7) contains a functional cytosolic adenylate cyclase catalytic centre. FEBS Lett. 2015, 589, 3848-3852. [CrossRef]

106. Al-Younis, I.; Wong, A.; Lemtiri-Chlieh, F.; Schmöckel, S.; Tester, M.; Gehring, C.; Donaldson, L. The Arabidopsis thaliana K ${ }^{+}-U_{\text {ptake }}$ Permease 5 (AtKUP5) contains a functional cytosolic adenylate cyclase essential for $\mathrm{K}^{+}$transport. Front. Plant Sci. 2018, 9, 1645. [CrossRef] 
107. Bianchet, C.; Wong, A.; Quaglia, M.; Alqurashi, M.; Gehring, C.; Ntoukakis, V.; Pasqualini, S. An Arabidopsis thaliana leucine-rich repeat protein harbors an adenylyl cyclase catalytic center and affects responses to pathogens. J. Plant Physiol. 2019, $232,12-22$. [CrossRef]

108. Ludidi, N.; Gehring, C. Identification of a novel protein with guanylyl cyclase activity in Arabidopsis thaliana. J. Biol. Chem. 2003, 278, 6490-6494. [CrossRef]

109. Mulaudzi, T.; Ludidi, N.; Ruzvidzo, O.; Morse, M.; Hendricks, N.; Iwuoha, E.; Gehring, C. Identification of a novel Arabidopsis thaliana nitric oxide-binding molecule with guanylate cyclase activity in vitro. FEBS Lett. 2011, 585, 2693-2697. [CrossRef]

110. Kwezi, L.; Ruzvidzo, O.; Wheeler, J.I.; Govender, K.; Iacuone, S.; Thompson, P.E.; Gehring, C.; Irving, H.R. The phytosulfokine (PSK) receptor is capable of guanylate cyclase activity and enabling cyclic GMP-dependent signaling in plants. J. Biol. Chem. 2011, 286, 22580-22588. [CrossRef]

111. Kwezi, L.; Meier, S.; Mungur, L.; Ruzvidzo, O.; Irving, H.; Gehring, C. The Arabidopsis thaliana Brassinosteroid Receptor (AtBRI1) contains a domain that functions as a guanylyl cyclase in vitro. PLoS ONE 2007, 2, e449. [CrossRef] [PubMed]

112. Meier, S.; Ruzvidzo, O.; Morse, M.; Donaldson, L.; Kwezi, L.; Gehring, C. The Arabidopsis Wall Associated Kinase-Like 10 gene encodes a functional guanylyl cyclase and is co-expressed with pathogen defense related genes. PLoS ONE 2010, 5, e8904. [CrossRef] [PubMed]

113. Rahman, H.; Wang, X.-Y.; Xu, Y.-P.; He, Y.-H.; Cai, X.-Z. Characterization of tomato protein kinases embedding guanylate cyclase catalytic center motif. Sci. Rep. 2020, 10, 1-16. [CrossRef] [PubMed]

114. Muleya, V.; Wheeler, J.I.; Ruzvidzo, O.; Freihat, L.; Manallack, D.T.; Gehring, C.; Irving, H.R. Calcium is the switch in the moonlighting dual function of the ligand-activated receptor kinase phytosulfokine receptor 1. Cell Commun. Signal. 2014, 12, 60. [CrossRef] [PubMed]

115. Sun, X.; Qiu, Y.; Peng, Y.; Ning, J.; Song, G.; Yang, Y.; Deng, M.; Men, Y.; Zhao, X.; Wang, Y.; et al. Close temporal relationship between oscillating cytosolic $\mathrm{K}^{+}$and growth in root hairs of Arabidopsis. Int. J. Mol. Sci. 2020, 21, 6184. [CrossRef]

116. Ahn, S.J.; Shin, R.; Schachtman, D.P. Expression of KT/KUP genes in Arabidopsis and the role of root hairs in $\mathrm{K}^{+}$uptake. Plant Physiol. 2004, 134, 1135-1145. [CrossRef]

117. Han, M.; Wu, W.; Wu, W.-H.; Wang, Y. Potassium transporter KUP7 is involved in $\mathrm{K}^{+}$acquisition and translocation in Arabidopsis root under $\mathrm{K}^{+}$-limited conditions. Mol. Plant 2016, 9, 437-446. [CrossRef]

118. Donaldson, L.; Ludidi, N.; Knight, M.R.; Gehring, C.; Denby, K. Salt and osmotic stress cause rapid increases in Arabidopsis thaliana cGMP levels. FEBS Lett. 2004, 569, 317-320. [CrossRef]

119. Shabala, S.; Wu, H.; Bose, J. Salt stress sensing and early signalling events in plant roots: Current knowledge and hypothesis. Plant Sci. 2015, 241, 109-119. [CrossRef]

120. Maathuis, F.J.M.; Sanders, D. Sodium uptake in Arabidopsis roots is regulated by cyclic nucleotides. Plant Physiol. 2001, 127, 1617-1625. [CrossRef]

121. Demidchik, V.; Tester, M. Sodium fluxes through nonselective cation channels in the plasma membrane of protoplasts from Arabidopsis roots. Plant Physiol. 2002, 128, 379-387. [CrossRef] [PubMed]

122. Demidchik, V.; Shabala, S.; Isayenkov, S.; Cuin, T.A.; Pottosin, I. Calcium transport across plant membranes: Mechanisms and functions. New Phytol. 2018, 220, 49-69. [CrossRef] [PubMed]

123. Demidchik, V.; Bowen, H.C.; Maathuis, F.J.M.; Shabala, S.N.; Tester, M.A.; White, P.J.; Davies, J.M. Arabidopsis thaliana root non-selective cation channels mediate calcium uptake and are involved in growth. Plant J. 2002, 32, 799-808. [CrossRef] [PubMed]

124. Kiegle, E.; Moore, C.A.; Haseloff, J.; Tester, M.A.; Knight, M.R. Cell-type-specific calcium responses to drought, salt and cold in the Arabidopsis root. Plant J. 2000, 23, 267-278. [CrossRef] [PubMed]

125. Niu, W.-T.; Han, X.-W.; Wei, S.-S.; Shang, Z.-L.; Wang, J.; Yang, D.-W.; Fan, X.; Gao, F.; Zheng, S.-Z.; Bai, J.-T.; et al. Arabidopsis cyclic nucleotide-gated channel 6 is negatively modulated by multiple calmodulin isoforms during heat shock. J. Exp. Bot. 2020, 71, 90-104. [CrossRef] [PubMed]

126. Lu, D.; Wu, S.; Gao, X.; Zhang, Y.; Shan, L.; He, P. A receptor-like cytoplasmic kinase, BIK1, associates with a flagellin receptor complex to initiate plant innate immunity. Proc. Natl. Acad. Sci. USA 2010, 107, 496-501. [CrossRef] [PubMed]

127. Gómez-Gómez, L.; Boller, T. FLS2: An LRR receptor-like kinase involved in the perception of the bacterial elicitor flagellin in Arabidopsis. Mol. Cell 2000, 5, 1003-1011. [CrossRef]

128. Ma, Y.; Walker, R.K.; Zhao, Y.; Berkowitz, G.A. Linking ligand perception by PEPR pattern recognition receptors to cytosolic Ca ${ }^{2+}$ elevation and downstream immune signaling in plants. Proc. Natl. Acad. Sci. USA 2012, 109, 19852-19857. [CrossRef]

129. Jeworutzki, E.; Roelfsema, M.R.G.; Anschütz, U.; Krol, E.; Elzenga, J.T.M.; Felix, G.; Boller, T.; Hedrich, R.; Becker, D. Early signaling through the Arabidopsis pattern recognition receptors FLS2 and EFR involves $\mathrm{Ca}^{2+}$-associated opening of plasma membrane anion channels. Plant J. 2010, 62, 367-378. [CrossRef]

130. Wang, F.-Z.; Zhang, N.; Guo, Y.-J.; Gong, B.-Q.; Li, J.-F. Split nano luciferase complementation for probing protein-protein interactions in plant cells. J. Integr. Plant Biol. 2020, 62, 1065-1079. [CrossRef]

131. Thor, K.; Peiter, E. Cytosolic calcium signals elicited by the pathogen-associated molecular pattern flg22 in stomatal guard cells are of an oscillatory nature. New Phytol. 2014, 204, 873-881. [CrossRef] [PubMed]

132. Thor, K.; Jiang, S.; Michard, E.; George, J.; Scherzer, S.; Huang, S.; Dindas, J.; Derbyshire, P.; Leitão, N.; DeFalco, T.A.; et al. The calcium-permeable channel OSCA1.3 regulates plant stomatal immunity. Nature 2020, 585, 569-573. [CrossRef] [PubMed] 
133. Zhang, X.-R.; Xu, Y.-P.; Cai, X.-Z. SlCNGC1 and SlCNGC14 suppress Xanthomonas oryzae pv. oryzicola-induced hypersensitive response and non-host resistance in tomato. Front. Plant Sci. 2018, 9, 9. [CrossRef]

134. Edel, K.H.; Marchadier, E.; Brownlee, C.; Kudla, J.; Hetherington, A.M. The evolution of calcium-based signalling in plants. Curr. Biol. 2017, 27, 667-679. [CrossRef] [PubMed]

135. O'Halloran, D.M.; Altshuler-Keylin, S.; Zhang, X.-D.; He, C.; Morales-Phan, C.; Yu, Y.; Kaye, J.A.; Brueggemann, C.; Chen, T.-Y.; L'Etoile, N.D. Contribution of the cyclic nucleotide gated channel subunit, CNG-3, to olfactory plasticity in Caenorhabditis elegans. Sci. Rep. 2017, 7, 169. [CrossRef]

136. Wilson, C.M.; Stecyk, J.A.W.; Couturier, C.S.; Nilsson, G.E.; Farrell, A.P. Phylogeny and effects of anoxia on hyperpolarizationactivated cyclic nucleotide-gated channel gene expression in the heart of a primitive chordate, the Pacific hagfish (Eptatretus stoutii). J. Exp. Biol. 2013, 216, 4462-4472. [CrossRef]

137. Chiasson, D.M.; Haage, K.; Sollweck, K.; Brachmann, A.; Dietrich, P.; Parniske, M. A quantitative hypermorphic CNGC allele confers ectopic calcium flux and impairs cellular development. eLife 2017, 6. [CrossRef]

138. Lelle, M.; Otte, M.; Bonus, M.; Gohlke, H.; Benndorf, K. Fluorophore-labeled cyclic nucleotides as potent agonists of cyclic nucleotide-regulated ion channels. ChemBioChem 2020, 21, 2311-2320. [CrossRef] 